\title{
Equivalence of Quantum Heat Machines, and Quantum-Thermodynamic Signatures
}

\author{
Raam Uzdin, ${ }^{*}$ Amikam Levy, and Ronnie Kosloff \\ Fritz Haber Research Center for Molecular Dynamics, \\ Hebrew University of Jerusalem, Jerusalem 91904, Israel \\ (Received 14 May 2015; published 29 September 2015)
}

\begin{abstract}
Quantum heat engines (QHE) are thermal machines where the working substance is a quantum object. In the extreme case, the working medium can be a single particle or a few-level quantum system. The study of QHE has shown a remarkable similarity with macroscopic thermodynamical results, thus raising the issue of what is quantum in quantum thermodynamics. Our main result is the thermodynamical equivalence of all engine types in the quantum regime of small action with respect to Planck's constant. They have the same power, the same heat, and the same efficiency, and they even have the same relaxation rates and relaxation modes. Furthermore, it is shown that QHE have quantum-thermodynamic signature; i.e., thermodynamic measurements can confirm the presence of quantum effects in the device. We identify generic coherent and stochastic work extraction mechanisms and show that coherence enables power outputs that greatly exceed the power of stochastic (dephased) engines.
\end{abstract}

DOI: 10.1103/PhysRevX.5.031044

\section{INTRODUCTION}

Thermodynamics emerged as a practical theory for evaluating the performance of steam engines. Since then, the theory proliferated and has been utilized in countless systems and applications. Eventually, thermodynamics became one of the pillars of theoretical physics. Amazingly, it survived great scientific revolutions such as quantum mechanics and general relativity. To a certain extent, thermodynamics even contributed to these theories (e.g., black hole entropy and temperature).

Despite its success, it is not expected that thermodynamics will hold all the way to the atomic scale, where the number of particles in the relevant substance is small or even equal to 1 . Thus, it was anticipated that in the quantum regime new thermodynamic effects will surface. However, quantum-thermodynamic systems (even with a single particle) show a remarkable similarity to the macroscopic system described by classical thermodynamic. When the baths are thermal, the Carnot efficiency limit is equally applicable for a small quantum system [1,2]. Even classical fluctuation theorems hold without any alteration [3-5].

Since real engines have a finite cycle time, they cannot be in an exact equilibrium state and perform as a reversible machine. Consequently, the efficiency is always lower than the Carnot limit. Furthermore, the performance of a real engine is more severely limited by heat leaks, friction, and

*raam@mail.huji.ac.il

Published by the American Physical Society under the terms of the Creative Commons Attribution 3.0 License. Further distribution of this work must maintain attribution to the author(s) and the published article's title, journal citation, and DOI.
Subject Areas: Quantum Physics

heat transport. This led to the study of efficiency at maximal power [6-9] and finite-time thermodynamics $[10,11]$. In analogy to the classical case, nonadiabatic couplings in finite-time quantum evolution give rise to a new quantum frictionlike mechanism [12,13]. However, this friction effect is not a generic feature of quantum heat machines. It can be avoided by applying different schemes. (See Ref. [14], or the multilevel embedding scheme in Sec. II E of this paper. See also the discussion in Ref. [15].)

Is there really nothing generic, new, and profound in the thermodynamics of small quantum system? Can classical thermodynamics and stochastic analysis predict and explain any observed thermodynamic effect in quantum heat machines? Do quantum effects always lead to friction and losses, or can they boost the heat machine performance? In this work, we present a generic thermodynamic behavior that is purely quantum in its essence and has no classical counterpart. Furthermore, it is shown that in the quantum regime, the generic coherent work extraction mechanism can significantly outperform the stochastic work extraction mechanism.

Quantum thermodynamics is the study of thermodynamic quantities such as temperature, heat, work, and entropy in microscopic quantum systems or even for a single particle. This study includes dynamical analysis of engines and refrigerators in the quantum regime $[1,14,16-40]$, theoretical frameworks that take into account single-shot events [41,42], and the study of thermalization mechanisms [43-45]. Another topic of interest in quantum thermodynamics is algorithmic cooling [46-50]. For updated reviews on quantum thermodynamics, we recommend Refs. [51,52].

Several proposals for quantum-heat-engine realization and experimental setup have been studied [53-57]. 
However, as will be explained later, only some of them are suited for exploring the quantum effects studied here. We hope our findings will motivate experimentalists to come up with more schemes that can probe the coherent work extraction regime.

Recently, some progress on the role of quantum coherence in quantum thermodynamics has been made [58-64]. In addition, quantum coherence has been shown to quantitatively affect the performance of heat machines [65-67]. In this work, we associate coherence with a specific thermodynamic effect and relate it to a thermodynamic work extraction mechanism.

Heat engines can be classified by their different scheduling of the interactions with the baths and the work repository. These types include the four-stroke, two-stroke, and the continuous engines (these engine types will be described in more detail later). The choice of engine type is usually guided by convenience of analysis or ease of implementation. Nevertheless, from a theoretical point of view, the fundamental differences or similarities between the various engine types are still uncharted. This is particularly true in the microscopic quantum regime. For brevity, we discuss engines, but all our results are equally applicable to other heat machines such as refrigerators and heaters.

Our first result (21)-(23) is that all three engine types are thermodynamically equivalent in the limit of small engine action (weak thermalization and a weak driving field). The equivalence holds also for transients and for states that are very far from thermal equilibrium. On top of providing a thermodynamic unification limit for the various engine types, this equivalence also establishes a connection to quantum mechanics, as it crucially depends on phase coherence and quantum interference. In particular, the validity regime of the equivalence is expressed in terms of $\hbar$.

Our second result (32) is the identification of a quantumthermodynamic signatures. Let us define a quantum signature as a signal extracted from measurements that unambiguously indicates the presence of quantum effects (e.g., entanglement or interference). The Bell inequality for the EPR experiment is a good example. A quantumthermodynamic signature is a quantum signature obtained from measuring thermodynamic quantities. We show that it is possible to set an upper bound on the work output of a stochastic, coherence-free engine. Any engine that surpasses this bound must have some level of coherence. Hence, work exceeding the stochastic bound constitutes a quantum-thermodynamic signature. Furthermore, we distinguish between a coherent work extraction mechanism and a stochastic work extraction mechanism. This explains why in the equivalence regime, coherent engines produce significantly more power compared to the corresponding stochastic engine. We estimate that our findings can be verified with present-day experimental capabilities. For a suggested realization in solid-state superconducting qubits, see Ref. [68].

The equivalence derivation is based on three ingredients. First, we introduce a multilevel embedding framework that enables the analysis of all three types of engines in the same physical setup. Next, a "norm action" smallness parameter, $s$, is defined for engines using Liouville space. The third ingredient is the symmetric rearrangement theorem that is used to show why all three engine types have the same thermodynamic properties despite the fact that they exhibit very different density matrix dynamics.

In Sec. II, we describe the main engine types and introduce the multilevel embedding framework. Next, in Sec. III, the multilevel embedding and the symmetric rearrangement theorem are used to show the various equivalence relation of different engine types. After discussing the two fundamental work extraction mechanisms, in Sec. IV, we present a quantum-thermodynamic signature that separates quantum engines from stochastic engines. In Sec. V, the over-thermalization effect in coherent quantum heat engines is studied. Finally, in Sec. VI, we conclude and discuss extensions and future prospects.

\section{HEAT ENGINES TYPES AND THE MULTILEVEL EMBEDDING SCHEME}

Heat engines are either discrete or continuous. Discrete engines include the two-stroke and four-stroke engines, whereas a turbine is a continuous engine [69]. These engine types appear in the macroscopic world as well as in the microscopic (quantum) realm. Here, we present a theoretical framework where all three types of engines can be embedded in a unified physical framework. This framework, termed "multilevel embedding," is an essential ingredient in our theory as it enables a meaningful comparison between different engine types.

\section{A. Heat and work}

A heat engine is a device that uses at least two thermal baths in different temperatures to extract work. Work is the transfer of energy from the engine to some external repository without changing the entropy of the repository. For example, increasing the excitation number of an oscillator, increasing the photon number in a specific optical mode (lasing), or increasing the kinetic energy in a single predefined direction. "Battery" or "flywheel" are terms often used in this context of work storage [70,71]. We shall use the more general term "work repository." Heat, on the other hand, is an energy exchange between the system and a thermal bath that involves entropy change in the bath. In the weak system-bath coupling limit, the heat is related to the temperature, via the well-known relation $d Q=T d S$, where $d S$ is the entropy change in the bath.

In the elementary quantum heat engines, the working substance is comprised of single particle (or a few at the 
most). Thus, the working substance cannot reach equilibrium on its own. Furthermore, excluding a few nongeneric cases, it is not possible to assign an equation of state that establishes a relation between thermodynamic quantities. Nevertheless, QHE's satisfy the second law and therefore are also bounded by the Carnot efficiency limit $[1,72]$.

Work strokes are characterized by zero contact with the baths and an inherently time-dependent Hamiltonian. The unitary evolution generated by this Hamiltonian can change the energy of the system. On the other hand, the von Neumann entropy and the purity remain fixed (unitary evolution at this stage). Hence, the energy change of the system in this case constitutes pure work. The system's energy change is actually an energy exchange with the work repository.

When the system is coupled to a thermal bath and the Hamiltonian is fixed in time, the bath can change the populations of the energy levels. In a steady state, the system reaches a Gibbs state where the density matrix has no coherences in the energy basis and the population of the levels is given by $p_{n, b}=e^{-\left(E_{n} / T_{b}\right)} / \sum_{n=1}^{N} e^{-\left(E_{n} / T_{b}\right)}$, where $N$ is the number of levels and " $b$ " stands for " $c$ " (cold) or " $h$ " (hot). In physical models where the system thermalizes via collision with bath particles, a full thermalization can be achieved in finite time [15,73-76]. However, it is not necessary that the baths will bring the system close to a Gibbs state for the proper operation of the engine. In particular, maximal efficiency (e.g., in Otto engines) can be achieved without full thermalization. Maximal power (work per cycle time) is also associated with partial thermalization $[6,8]$. The definitive property of a thermal bath is its aspiration to bring the system to a predefined temperature regardless of the initial state of the system. The evolution in this stage does not conserve the eigenvalues of the density matrix of the system, and therefore, not only energy but entropy as well is exchanged with the bath. Therefore, the energy exchange in this stage is considered as heat.

In contrast to definitions of heat and work that are based on the derivative of the internal energy [1,77,78], our definitions are obtained by energy balance when coupling only one element (bath or external field) at a time. As we see later, in some engine types, several agents change the internal energy simultaneously. Even in this case, this point of view of heat and work will still be useful for obtaining consistent and physical definitions of heat and work.

\section{B. Three types of engines}

There are three core engine types that operate with two thermal baths: four-stroke engine, two-stroke engine, and a continuous engine. A stroke is a time segment where a certain operation takes place, for example, thermalization or work extraction. By definition, adjacent strokes in heat engines do not commute with each other. If they do commute (for example, see the "cold" and "hot" operations in the two-stroke engine later), they can be combined into a single stroke since the total effect of the two strokes can be generated by applying the two operations simultaneously.

Each stroke is a completely positive $(C P)$ map [79], and therefore, the one-cycle evolution operator of the engine is also a $C P$ map. For the extraction of work, it is imperative that some of the stroke propagators do not commute [80].

Otto engines and Carnot engines are examples of fourstroke engines. The simplest quantum four-stroke engine is the two-level Otto engine shown in Fig. 1(a). In the first stroke, only the cold bath is connected to the system. Thus, the internal energy changes are associated with heat exchange with the cold bath. The expansion and compression of the levels are fully described by a time-dependent Hamiltonian of the form $H(t)=f(t) \sigma_{z}$ (the baths are disconnected at this stage). In the second stroke, work is consumed in order to expand the levels, and in the fourth stroke, work is produced when levels revert to their original values. There is a net work extraction since the populations in stages II and IV are different. In different engines, much more general unitary transformation can be used to extract work. Nevertheless, this particular operation resembles the classical expansion and compression of classical engines. The work is the energy exchanged with the system during the unitary stages: $W=W_{I I}+W_{I V}=\left(\left\langle E_{3}\right\rangle-\left\langle E_{2}\right\rangle\right)+$ $\left(\left\langle E_{5}\right\rangle-\left\langle E_{4}\right\rangle\right)$. We consider only energy expectation values for two main reasons. First, investigations of work fluctuations revealed that quantum heat engines follow classical fluctuation laws [72], and we search for quantum signatures in heat engines. The second reason is that, in our view, the engine should not be measured during operation. The measurement protocol used in quantum fluctuation theorems $[3,4,72]$ eliminates the density-matrix coherences. These coherences have a critical component in

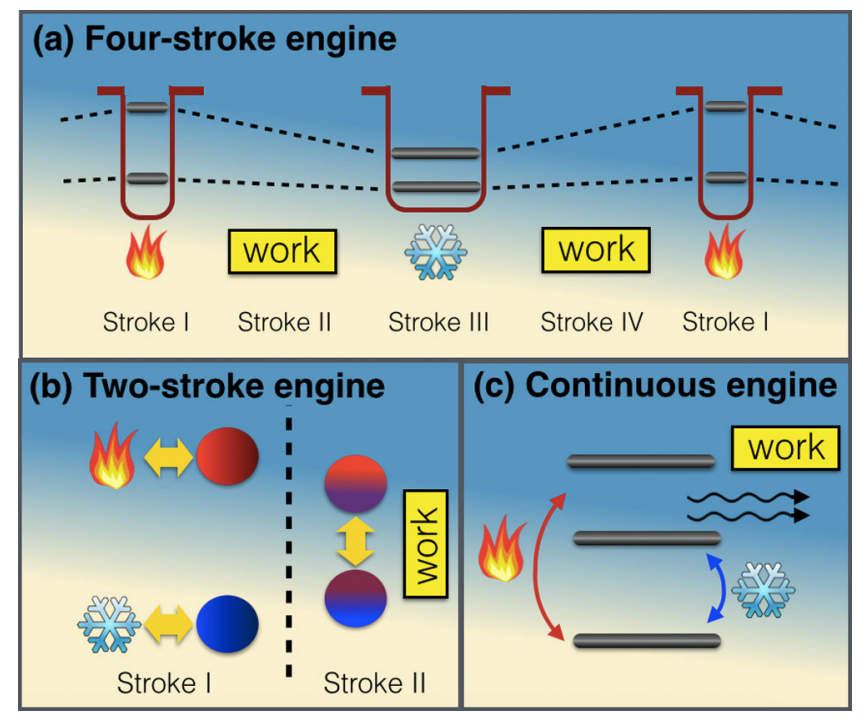

FIG. 1. (a) A two-level scheme of a four-stroke engine. (b) A two-particle scheme of a two-stroke engine. (c) A three-level scheme of a continuous engine. 
the equivalence and quantum signature we study in this paper. As shown in Sec. IV, measurements or dephasing dramatically change the engine. Thus, although we frequently calculate work per cycle, the measured quantity is the cumulative work, and it is measured only at the end of the process. The averaged quantities are obtained by repeating the full experiment many times. Engines are designed to perform a task, and we assume that this completed task is the subject of measurement. The engine internal state is not measured.

The heat per cycle taken from the cold bath is $Q_{c}=\left\langle E_{2}\right\rangle-\left\langle E_{1}\right\rangle$, and the heat taken from the hot bath is $Q_{h}=\left\langle E_{4}\right\rangle-\left\langle E_{3}\right\rangle$. In a steady state, the average energy of the system returns to its initial value after one cycle [81] so that $\left\langle E_{5}\right\rangle=\left\langle E_{1}\right\rangle$. From this result, it follows immediately that $Q_{c}+Q_{h}+W=0$; i.e., the first law of thermodynamics is obeyed. There is no instantaneous energy conservation of internal energy, as energy may be temporarily stored in the interaction field or in the work repository.

In the two-stroke engine shown in Fig 1(b), the engine consists of two parts (e.g., two qubits) [82]. One part may couple only to the hot bath, and the other may couple only to the cold bath. In the first stroke, both parts interact with their bath (but do not necessarily reach equilibrium). In the second unitary stroke, the two engine parts are disconnected from the baths and are coupled to each other. They undergo a mutual unitary evolution, and work is extracted in the process.

In the continuous engine shown in Fig. 1(c), the two baths and the external interaction field are connected continuously. For example, in the three-level laser system shown in Fig 1(c), the laser light represented by $\mathcal{H}_{w}(t)$ generates stimulated emission that extracts work from the system. This system was first studied in a thermodynamics context in Ref. [83], while a more comprehensive dynamical analysis of the system was given in Ref. [84]. It is imperative that the external field is time dependent. If it is time independent, the problem becomes a pure heat transport problem where $Q_{h}=-Q_{c} \neq 0$. In heat transport, the interaction field merely "dresses" the level so that the baths see a slightly modified system. The Lindblad generators are modified accordingly, and heat flows without extracting or consuming work [85]. Variations on these engine types may emerge because of realization constraints. For example, in the two-stroke engine, the baths may be continuously connected. This variation and others can still be analyzed using the tools presented in this paper.

\section{Efficiency vs work and heat}

Since the early days of Carnot, efficiency received considerable attention for two main reasons. First, this quantity is of great interest from both theoretical and practical points of view. Second, unlike other thermodynamics quantities, the efficiency satisfies a universal bound that is independent of the engine details. The Carnot efficiency bound is a manifestation of the second law of thermodynamics. Indeed, for Markovian bath dynamics, it was shown that quantum heat engines cannot exceed the Carnot efficiency [1]. Recently, a more general approach based on a fluctuation theorem for QHE showed that the Carnot bound still holds for quantum engines [72]. Studies in which higher-than-Carnot efficiency are reported [66] are interesting, but they use nonthermal baths and therefore, not surprisingly, deviate from results derived in the thermodynamic framework that deals with thermal baths. For example, an electric engine is not limited to Carnot efficiency since its power source is not thermal. Although the present work has an impact on efficiency as well, we focus on work and heat separately in order to unravel quantum effects. As will be exemplified later, in some elementary cases, these quantum effects do not influence the efficiency.

\section{Bath description and Liouville space}

The dynamics of the working fluid (system) interacting with the heat baths is described by the Lindblad-GoriniKossakowski-Sudarshan (LGKS) master equation for the density matrix $[79,86,87]$ :

$\hbar d_{t} \rho=L(\rho)=-i\left[H_{s}, \rho\right]+\sum_{k} A_{k} \rho A_{k}^{\dagger}-\frac{1}{2} A_{k}^{\dagger} A_{k} \rho-\frac{1}{2} \rho A_{k}^{\dagger} A_{k}$,

where the $A_{k}$ operators depend on the temperature, relaxation time of the bath, system bath coupling, and also on the system Hamiltonian $H_{s}$ [79]. This form already encapsulates within the Markovian assumption of no bath memory. The justification for these equations arises from a "microscopic derivation" in the weak system-bath coupling limit [88]. In this derivation, a weak interaction field couples the system of interest to a large system (the bath) with temperature $T$. This interaction brings the system into a Gibbs state at temperature $T$. The Lindblad thermalization operators $A_{k}$ used for the baths are described in the next section. The small Lamb shift is ignored.

Equation (1) is a linear equation, so it can always be rearranged into a vector equation. Given an index mapping $\rho_{N \times N} \rightarrow|\rho\rangle_{1 \times N^{2}}$, the Lindblad equation now reads

$$
i \hbar d_{t}|\rho\rangle=\left(\mathcal{H}_{H}+\mathcal{L}\right)|\rho\rangle \doteq \mathcal{H}|\rho\rangle,
$$

where $\mathcal{H}_{H}$ is a Hermitian $N^{2} \times N^{2}$ matrix that originates from $H_{s}$, and $\mathcal{L}$ is a non-Hermitian $N^{2} \times N^{2}$ matrix that originates from the Lindblad evolution generators $A_{k}$. This extended space is called Liouville space [89]. In this paper, we use calligraphic letters to describe operators in Liouville space and ordinary letters for operators in Hilbert space. For states, however, $|A\rangle$ will denote a vector in Liouville space formed from $A_{N \times N}$ by "vec-ing" $A$ into a column in the same procedure $\rho$ is converted into $|\rho\rangle$. A short review 
of Liouville space and some of its properties is given in Appendix A.

In unitary dynamics, the largest energy gap of the Hamiltonian sets a speed limit on the rate of change of a state (e.g., rotation speed in the Bloch sphere). Since $\mathcal{H}$ is not Hermitian, the energy scalar that sets a speed limit on the evolution speed is the spectral norm (or operator norm) of $\mathcal{H},\|\mathcal{H}\|=\max \sqrt{\operatorname{eig}\left(\mathcal{H}^{\dagger} \mathcal{H}\right)}$ (The spectral norm is the largest singular value of $\mathcal{H}[90])$. In particular, we show in Appendix B that the norm action, defined as

$$
s=\int_{0}^{\tau}\|\mathcal{H}(t)\| d t,
$$

sets a limit on how much a state can change during a time $\tau$ because of the operation of $\mathcal{H}$. For time-independent superHamiltonian $\mathcal{H}$, the evolution operator in Liouville space is

$$
|\rho(t)\rangle=\mathcal{K}\left|\rho\left(t^{\prime}\right)\right\rangle=e^{-i \mathcal{H}\left(t-t^{\prime}\right) / \hbar}\left|\rho\left(t^{\prime}\right)\right\rangle .
$$

Writing the evolution operator as an exponent of a matrix has a significant advantage since commutator exponentiation is avoided. Furthermore, the action has a natural definition in this formalism. In principal, it should be possible to reformulate the derivations using density matrixes and the Kraus operators. However, it seems that the Hilbert space formalism is far more cumbersome and complicated (for example, see Refs. [91,92]).

While the Lindblad description works very well for sufficiently long times, it fails for very short times where some of the approximation breaks down. In scales where the bath still has a memory of the system's past states, the semigroup property of the Lindblad equation no longer holds: $\left|\rho\left(t+t^{\prime}\right)\right\rangle \neq e^{-i\left(\mathcal{H}_{s}+\mathcal{L}\right)\left(t-t^{\prime}\right) / \hbar}\left|\rho\left(t^{\prime}\right)\right\rangle$. This will set a cutoff limit for the validity of the engine-type equivalence in the Markovian approximation.

Next we introduce the multilevel embedding scheme that enables us to discuss various heat engines in the same physical setup.

\section{E. Multilevel embedding}

Let the working substance of the quantum engine be an $N$-level system. These levels are fixed in time [i.e., they do not change as in Fig. 1(a)]. For simplicity, the levels are assumed to be nondegenerate. We divide the energy levels into a cold manifold and a hot manifold. During the operation of the engine, the levels in the cold manifold interact only with the cold bath, and the levels in the hot manifold interact only with the hot bath. Each thermal coupling can be turned on and off as a function of time, but the aliasing of a level to a manifold does not change in time.

If the manifolds do not overlap, the hot and cold thermal operations commute and they can be applied at the same time or one after the other. The end result will be the same.
Nevertheless, our scheme also includes the possibility that one level appears in both manifolds. This is the case for the three-level continuous engine shown in Fig. 1(c). For simplicity, we exclude the possibility of more than one mutual level. If there are two or more overlapping levels, there is an inevitable heat transport in the steady state from the hot bath to the cold bath even in the absence of an external field that extracts work. In the context of heat engines, this can be interpreted as heat leak. This "no fieldno transport" condition holds for many engines studied in the literature. Nonetheless, this condition is not a necessary condition for the validity of our results.

This manifold division seems sensible for the continuous engine and even for the two-stroke engine in Fig. 1(b), but how can it be applied to the four-stroke engine shown in Fig. 1(a)? The two levels interact with both baths and also change their energy value in time, contrary to the assumption of fixed energy levels. Nevertheless, this engine is also incorporated into the multilevel embedding framework. Instead of two levels as in Fig. 1(a), consider the four-level system shown in the dashed green lines in Fig. 2.

Initially, only levels 2 and 3 are populated and coupled to the cold bath ( 2 and 3 are in the cold manifold). In the unitary stage, an interaction Hamiltonian $H_{\text {swap }}$ generates a full swap of populations and coherence according to the rule $1 \leftrightarrow 2,3 \leftrightarrow 4$. Now, levels 1 and 4 are populated and 2 and 3 are empty. Therefore, this system fully simulates the expanding-level engine shown in Fig. 1(a). At the same time, this system satisfies the separation into well-defined

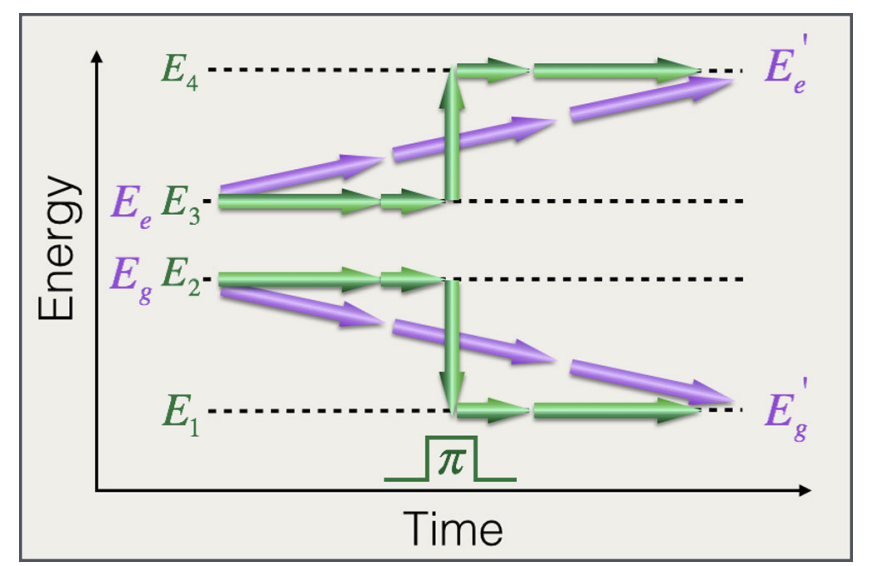

FIG. 2. In the standard two-level Otto engine, there are twolevel $E_{g, e}$ (purple arrows) that change in time to $E_{g, e}^{\prime}$. In the multilevel embedding framework, the levels $\left(E_{1-4}\right)$ are fixed in time (black dashed lines), but a time-dependent field ( $\pi$ pulse, swap operation) transfers the population (green arrows) to the other levels. For a swap operation, the two schemes lead to the same final state and therefore are associate with the same work. Nonetheless, the multilevel scheme is more general since for weaker unitary transformation (instead of the $\pi$ pulse), coherences are generated. We show that this type of coherence can significantly boost the power output of the engine. 
time-independent manifolds, as defined in the multilevel embedding scheme.

The full swap used to embed the traditional four-stroke Otto engine is not mandatory, and other unitary operations can be applied. This extension of the four-stroke scheme is critical for our work since the equivalence of engines appears when the unitary operation is fairly close to the identity transformation. A full swap turns one diagonal state into another. Consequently, the steady state of an engine with full swap operation will not contain any coherences in the energy basis. As will be shown later, a partial swap or a different "weaker than full swap" unitary leads to steady-state coherences that dramatically enhance the power output. Note that these coherences between the hot and cold manifold imply a superposition of the cold and hot states. In other words, in contrast to the full swap case, the particle is not localized exclusively on either the hot or cold manifold.

Figures 3(a)-(c) show how the three types of engines are represented in the multilevel embedding scheme. The advantage of the multilevel scheme now becomes clear. All three engine types can be described in the same physical system with the same baths and the same coupling to external fields (work extraction). The engine types differ only in the order of the coupling to the baths and to the work repository. While the thermal operations commute if the manifolds do not overlap, the unitary operation never commutes with the thermal strokes.

In the present paper, we use a direct sum structure for the hot and cold manifolds. However, when there are two or more particles in the engine [82], it is more natural to apply a tensor product structure for the manifolds of the multilevel embedding scheme.

On the right of Fig. 3, we plotted a "brick" diagram for the evolution operator. Black stands for unitary transformation generated by some external field, while blue and red stand for hot and cold thermal coupling,

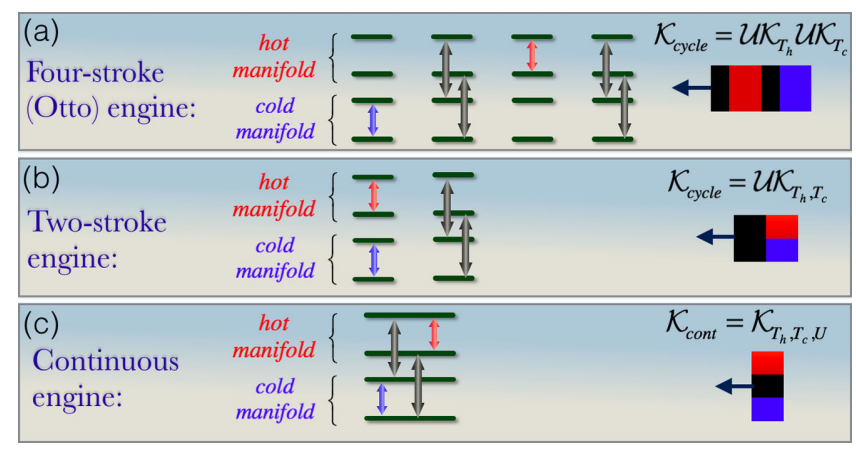

FIG. 3. Representation of the three types of engines (a)-(c) in the multilevel embedding framework. In this scheme, the different engine types differ only in the order of coupling to the baths and work repository. Since the interactions and energy levels are the same for all engine types, a meaningful comparison of performance becomes possible. respectively. When the bricks are on top of each other, it means that they operate simultaneously. Now we are in a position to derive the first main results of this paper: the thermodynamic equivalence of the different engine types in the quantum regime.

\section{CONTINUOUS AND STROKE ENGINE EQUIVALENCE}

We first discuss the equivalence of continuous and fourstroke engines. Nevertheless, all the arguments are valid for the two-stroke engines as well, as explained later. Although our results are not limited to a specific engine model, it will be useful to consider the simple engine shown in Fig. 4. We use this model to highlight a few points and also for numerical simulations. The Hamiltonian part of the system is

$$
H_{0}+\cos (\omega t) H_{w},
$$

where $H_{0}=-\left(\Delta E_{h} / 2\right)|1\rangle\left\langle 1\left|-\left(\Delta E_{c} / 2\right)\right| 2\right\rangle\langle 2|+\left(\Delta E_{c} /\right.$ $2)|3\rangle\left\langle 3\left|+\left(\Delta E_{h} / 2\right)\right| 4\right\rangle\left\langle 4\left|, H_{w}=\epsilon(t)\right| 1\right\rangle\langle 2|+\epsilon(t)| 3\rangle\langle 4|+$ H.c. and $\omega=\left(\Delta E_{h}-\Delta E_{c}\right) / 2 \hbar$.

The driving frequency that couples the system to the work repository is in resonance with the top and bottom energy gaps. The specific partitioning into hot and cold manifolds was chosen so that only one frequency (e.g., a single laser) is needed for implementing the system instead of two.

We assume that the Rabi frequency of the drive $\epsilon$ is smaller than the decay time scale of the baths, $\epsilon \ll \gamma_{c}, \gamma_{h}$. Under this assumption, the dressing effect of the driving field on the system-bath interaction can be ignored. It is justified, then, to use "local" Lindblad operators obtained in the absence of a driving field [85,93]. For plotting purposes (reasonable duty cycle), in the numerical examples, we often use $\epsilon=\gamma_{c}=\gamma_{h}$. While this poses no problem for stroke-engine realizations, for experimental demonstration of equivalence with continuous engines, one has to increase the duty cycle so that $\epsilon \ll \gamma_{c}, \gamma_{h}$. In other words, the unitary stage should be made longer but with a weaker driving field.

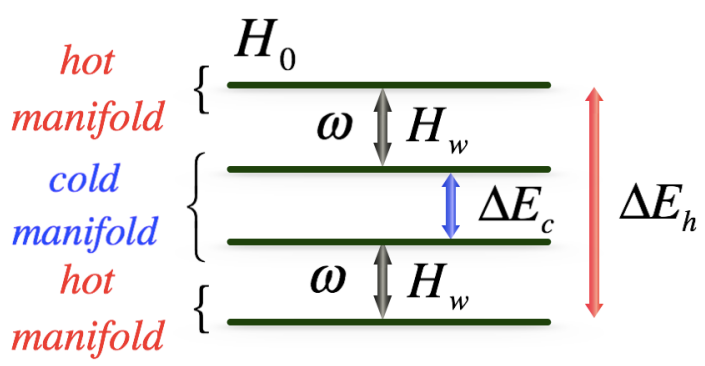

FIG. 4. Illustration of the engine used in the numerical simulation. By changing the time order of the coupling to $H_{w}$ and to thermal baths, all three types of engines can be realized in the model. 
The Lindblad equation is given by Eq. (1) with the Hamiltonian (5) and with the following Lindblad operators in Hilbert space:

$$
\begin{aligned}
& A_{1}=\sqrt{\gamma_{h}} e^{-\left(\Delta E_{h} / 2 T_{h}\right)}|4\rangle\langle 1|, \\
& A_{2}=\sqrt{\gamma_{h}}|1\rangle\langle 4|, \\
& A_{3}=\sqrt{\gamma_{c}} e^{-\left(\Delta E_{c} / 2 T_{c}\right)}|3\rangle\langle 2|, \\
& A_{4}=\sqrt{\gamma_{c}}|2\rangle\langle 3| .
\end{aligned}
$$

In all the numerical simulations, we use $\Delta E_{h}=4$, $\Delta E_{c}=1, T_{h}=5, T_{c}=1$. The interaction with the baths or with work repository can be turned on and off at will.

Starting with the continuous engine, we choose a unit cell that contains exactly $6 m$ ( $m$ is an integer) complete cycles of the drive $\left(\tau_{d}=2 \pi / \omega\right)$ so that $\tau_{\mathrm{cyc}}=6 m \tau_{d}$. The difference between the engine cycle time and the cycles of the external drive will become clear in stroke engines (also, the factor of 6 will be clarified).

For the validity of the secular approximation used in the Lindblad microscopic derivation [79], the evolution time scale must satisfy $\tau \gg(2 \pi \hbar) / \min \left(\Delta E_{h}, \Delta E_{c}\right)$. Therefore, $m$ must satisfy $m \gg(\hbar \omega) / \min \left(\Delta E_{h}, \Delta E_{c}\right)$. Note that if the Lindblad description is obtained from a different physical mechanism (e.g., thermalizing collisions), then this condition is not required.

Next, we transform to the interaction picture (denoted by tilde) using the transformation $\mathcal{U}=e^{-i \mathcal{H}_{0} t / \hbar}$, and perform the rotating wave approximation (RWA) by dropping terms oscillating at a frequency of $2 \omega$. For the RWA to be valid, the amplitude of the field must satisfy $\epsilon \ll \omega$. The resulting Liouville space super-Hamiltonian is

$$
\tilde{\mathcal{H}}=\mathcal{L}_{c}+\mathcal{L}_{h}+\frac{1}{2} \mathcal{H}_{w}
$$

Note that $\mathcal{L}_{h, c}$ were not modified by the transformation to the rotating system since $\left[\mathcal{L}_{h, c}, \mathcal{H}_{0}\right]=0$ in the microscopic derivation [94]. The oscillatory time dependence has disappeared because of the RWA and the interaction picture. There is still an implicit time dependence that determines which of the terms $\mathcal{L}_{c}, \mathcal{L}_{h}, \mathcal{H}_{w}$ is coupled to the system at a given time. We point out that when the RWA is not valid, the dynamics becomes considerably more complicated. First, even the basic unitary evolution has no simple analytical solution. Second, the Lindblad description of the continuous engine becomes more complicated. Thus, our analysis is restricted to the validity regime of the RWA.

The Lindblad Markovian dynamics and the RWA set a validity regime for our theory. This regime is the default regime used in quantum open systems (see Refs. [77,79]). It is intriguing to study how the results presented here are modified by the breakdown of the RWA or by bath memory effects. However, this analysis is beyond the scope of the present paper.

Now that we have established a regime of validity and the super-Hamiltonian that governs the system, we can turn to the task of transforming from one engine type to other types and study what properties change in this transformation. The engine-type transformation is based on the Strang decomposition [95-97] for two noncommuting operators $\mathcal{A}$ and $\mathcal{B}$ (the operators may not be Hermitian):

$$
e^{(\mathcal{A}+\mathcal{B}) d t}=e^{\frac{1}{2} \mathcal{A} d t} e^{\mathcal{B} d t} e^{\frac{1}{2} \mathcal{A} d t}+O\left[(s / \hbar)^{3}\right] \cong e^{\frac{1}{2} \mathcal{A} d t} e^{\mathcal{B} d t} e^{\frac{1}{2} \mathcal{A} d t},
$$

where the norm action (3), $s=(\|\mathcal{A}\|+\|\mathcal{B}\|) d t$, must be small for the expansion to be valid. $\|\mathcal{A}\|$ is the spectral norm of $\mathcal{A}$. In Appendix $\mathrm{C}$, we derive the condition $s \ll$ $\frac{1}{2} \hbar$ for the validity of Eq. (11). We use the symbol $\cong$ to denote equality with correction $O\left[(s / \hbar)^{3}\right]$.

Let the evolution operator of the continuous engine over the chosen cycle time $\tau_{\text {cyc }}=6 m \tau_{d}$ be

$$
\tilde{\mathcal{K}}^{\mathrm{cont}}=e^{-i \tilde{\mathcal{H}} \tau_{\mathrm{cyc}} / \hbar}
$$

By first splitting $\mathcal{L}_{c}$ and then splitting $\mathcal{L}_{h}$, we get

$$
\begin{aligned}
\tilde{\mathcal{K}}^{\text {four stroke }}= & e^{-i\left(3 \mathcal{L}_{c}\right)\left(\tau_{\text {cyc }} / 6 \hbar\right)} e^{-i\left(\frac{3}{2} \mathcal{H}_{w}\right)\left(\tau_{\text {cyc }} / 6 \hbar\right)} e^{-i\left(3 \mathcal{L}_{h}\right)\left(\tau_{\text {cyc }} / 3 \hbar\right)} \\
& \times e^{-i\left(\frac{3}{2} \mathcal{H}_{w}\right)\left(\tau_{\text {cyc }} / 6 \hbar\right)} e^{-i\left(3 \mathcal{L}_{c}\right)\left(\tau_{\mathrm{cyc}} / 6 \hbar\right)}
\end{aligned}
$$

Note that the system is periodic so the first and last stages are two parts of the same thermal stroke. Consequently, Eq. (13) describes an evolution operator of a four-stroke engine, where the unit cell is symmetric. This splitting is illustrated in Figs. 5(a) and 5(b). There are two thermal strokes and two work strokes that together constitute an evolution operator that describes a four-stroke engine. The cumulative evolution time as written above is $(m+m+2 m+m+m) \tau_{d}=6 m \tau_{d}=\tau_{\mathrm{cyc}}$. Yet, to maintain the same cycle time as chosen for the continuous engine, the coupling to the baths and field were multiplied by 3 . In this four-stroke engine, each thermal or work stroke operates, in total, only a third of the cycle time compared to the continuous engine. Hence, the coupling must be 3 times larger in order to generate the same evolution.

By virtue of the Strang decomposition, $\tilde{\mathcal{K}}^{\text {four stroke }} \cong$ $\tilde{\mathcal{K}}^{\text {cont }}$ if $s \ll \hbar$. The action parameter $s$ of the engine is defined as $s=\int_{-\tau_{\text {cyc }} / 2}^{\tau_{\text {cyc }} / 2}\|\tilde{\mathcal{H}}\| d t=\left(\frac{1}{2}\left\|\mathcal{H}_{w}\right\|+\left\|\mathcal{L}_{h}\right\|+\right.$ $\left.\left\|\mathcal{L}_{c}\right\|\right) \tau_{\text {cyc }}$. Note that the relation $\tilde{\mathcal{K}}^{\text {four stroke }} \cong \tilde{\mathcal{K}}^{\text {cont }}$ holds only when the engine action is small compared to $\hbar$. This first appearance of a quantum scale will be discussed later. 

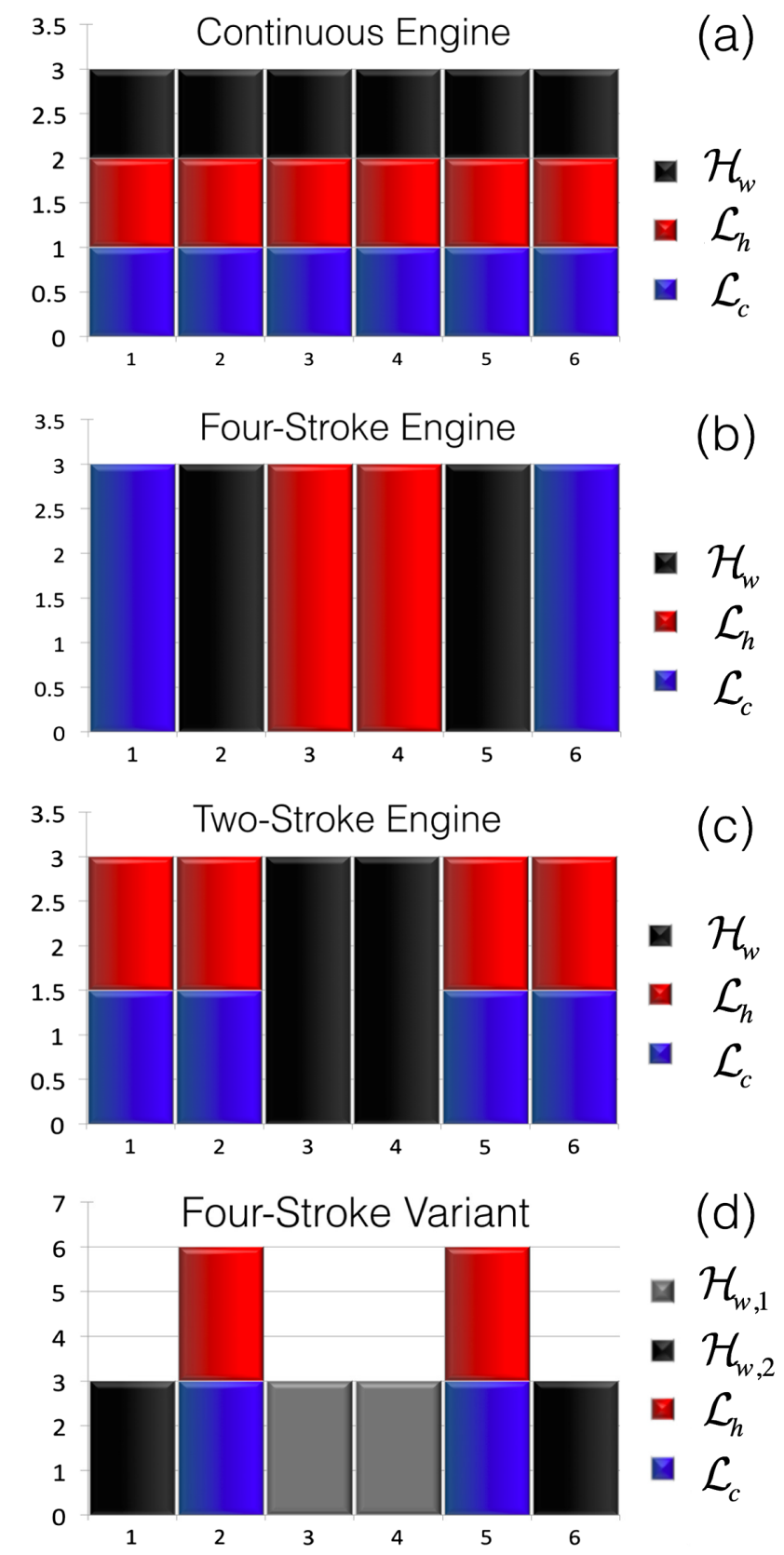

FIG. 5. Graphical illustrations of the super-Hamiltonians of various engines (a)-(d). The horizontal axis corresponds to time. The brick size corresponds to the strength of the coupling to the work repository or to the baths. The Hamiltonians are related to each other by applying the Strang decomposition to the evolution operators (12), (13), and (20). The symmetric rearrangement theorem ensures that in the limit of small action, any rearrangement that is symmetric with respect to the center and conserves the area of each color does not change the total power and heat over one cycle.

\section{A. Dynamical aspect of the equivalence}

The equivalence of the evolution operators $\tilde{\mathcal{K}}^{\text {four stroke }} \cong$ $\tilde{\mathcal{K}}^{\text {cont }}$ has two immediate important consequences. First, both engines have the same steady-state solution over one cycle $\left|\tilde{\rho}_{s}\right\rangle$ :

$$
\begin{gathered}
\tilde{\mathcal{K}}^{\text {four stroke }}\left(\tau_{\text {cyc }}\right)\left|\tilde{\rho}_{s}\right\rangle \cong \tilde{\mathcal{K}}^{\text {cont }}\left(\tau_{\text {cyc }}\right)\left|\tilde{\rho}_{s}\right\rangle=\left|\tilde{\rho}_{s}\right\rangle, \\
\left(\mathcal{L}_{c}+\mathcal{L}_{h}+\frac{1}{2} \mathcal{H}_{w}\right)\left|\tilde{\rho}_{s}\right\rangle=0
\end{gathered}
$$

At time instances that are not integer multiples of $\tau_{\text {cyc }}$, the states of the engines will differ significantly $\left(O\left[(s / \hbar)^{1}\right]\right)$ since $\quad \tilde{\mathcal{K}}^{\text {four stroke }}\left(t<\tau_{\text {cyc }}\right) \neq \tilde{\mathcal{K}}^{\text {cont }}\left(t<\tau_{\text {cyc }}\right)$. In other words, the engines are still significantly different from each other. The second consequence is that the two engines have the same transient modes as well. When monitored at multiples of $\tau_{\text {cyc }}$, both engines will have the same relaxation dynamics to the steady state if they started from the same initial condition. In the remainder of the paper, when the evolution operator is written without a time tag, this means that we are considering the evolution operator of a complete cycle.

We point out that there are higher-order decompositions where the correction terms are smaller than $O\left[(s / \hbar)^{3}\right]$. However, it turns out that these decompositions inherently involve negative coefficients [98]. A negative coefficient implies a thermal stroke of the form $e^{+i \mathcal{L} d t / \hbar}$ (instead of $\left.e^{-i \mathcal{L} d t / \hbar}\right)$. This type of evolution cannot be generated by a Markovian bath. Therefore, among the symmetric decompositions, the Strang decomposition seems to be the only one that can be used for decomposing Markovian thermal engine evolution operators.

\section{B. Thermodynamic aspect of the equivalence}

The equivalence of the one-cycle evolution operators of the two engines does not immediately imply that the engines are thermodynamically equivalent. Generally, in stroke engines, the heat and work depend on the dynamics of the state inside the cycle, which is very different $\left(O\left[(s / \hbar)^{1}\right]\right)$ from the constant state of the continuous engine. However, in this section, we show that all thermodynamics properties are equivalent in both engines up to $O\left[(s / \hbar)^{3}\right]$ corrections, similarly to the evolution operator. We start by evaluating the work and heat in the continuous engine. By considering infinitesimal time elements where $\mathcal{L}_{c}, \mathcal{L}_{h}$, and $\mathcal{H}_{w}$ operate separately, one obtains that the heat and work currents are $j_{c(h)}=\left\langle H_{0}\left|(1 / \hbar) \mathcal{L}_{c(h)}\right| \tilde{\rho}_{s}(t)\right\rangle$ and $j_{w}=\left\langle H_{0}\left|(1 / 2 \hbar) \mathcal{H}_{w}\right| \tilde{\rho}_{s}(t)\right\rangle$, where $\left\langle H_{0}|=| H_{0}\right\rangle^{\dagger}$ is the vectorized form of the field-free Hamiltonian $H_{0}$ of the system [see Eq. (5)]. See Appendix A for the use of bracket notation to describe expectation values $\langle A\rangle=\operatorname{tr}(A \rho)=$ $\langle A \mid \rho\rangle$. In principle, to calculate $\langle A\rangle$ in the rotating frame using $\left|\tilde{\rho}_{s}(t)\right\rangle,\langle A|$ must be rotated as well. However, because of the property $\left\langle H_{0}\right| \mathcal{H}_{0}=0$ shown in Appendix A, $\left\langle H_{0}\right|$ is not affected by this rotation.

In the continuous engine, the steady state satisfies $\left|\tilde{\rho}_{s}(t)\right\rangle=\left|\tilde{\rho}_{s}\right\rangle$, so the total heat and work in the steady state in one cycle are 


$$
\begin{aligned}
W^{\text {cont }} & =\left\langle H_{0}\left|\frac{1}{2 \hbar} \mathcal{H}_{w}\right| \tilde{\rho}_{s}\right\rangle \tau_{\mathrm{cyc}}, \\
Q_{c(h)}^{\mathrm{cont}} & =\left\langle H_{0}\left|\frac{1}{\hbar} \mathcal{L}_{c(h)}\right| \tilde{\rho}_{s}\right\rangle \tau_{\mathrm{cyc}} .
\end{aligned}
$$

These quantities should be compared to the work and heat in the four-stroke engine. Instead of carrying out the explicit calculation for this specific four-stroke splitting, we use the symmetric rearrangement theorem (SRT) derived in Appendix D. Symmetric rearrangement of a Hamiltonian is a change in the order of couplings $\epsilon(t), \gamma_{c}(t), \gamma_{h}(t)$ that satisfies $\int \epsilon(t) d t=$ const, $\int \gamma_{c}(t) d t=$ const, $\int \gamma_{h}(t) d t=$ const, and with the symmetry $\epsilon(t)=\epsilon(-t), \gamma_{c}(t)=\gamma_{c}(-t), \gamma_{c}(t)=\gamma_{c}(-t)$. Any super-Hamiltonian obtained using the Strang splitting of the continuous engine [for example, $\mathcal{H}^{\text {two stroke }}(t)$, $\left.\mathcal{H}^{\text {four stroke }}(t)\right]$ constitutes a symmetric rearrangement of the continuous engine. The SRT exploits the symmetry of the Hamiltonian to show that symmetric rearrangement changes heat and work only in $O\left[(s / \hbar)^{3}\right]$. In Appendix D, we show that

$$
\begin{aligned}
& W^{\text {four stroke }} \cong W^{\text {cont }}, \\
& Q_{c(h)}^{\text {four stroke }} \cong Q_{c(h)}^{\text {cont }} .
\end{aligned}
$$

Thus, we conclude that up to $(s / \hbar)^{3}$ corrections, the engines are thermodynamically equivalent. When $s \ll 1$, work, power, heat, and efficiency converge to the same value for all engine types. Clearly, inside the cycle, the work and heat in the two engines are significantly different $\left(O\left[(s / \hbar)^{1}\right]\right)$, but after a complete cycle, they become equivalent. The symmetry makes this equivalence more accurate as it holds up to $(s / \hbar)^{3}$ [rather than $(s / \hbar)^{2}$ ]. Interestingly, the work done in the first half of the cycle is $\frac{1}{2} W^{\text {cont }}+O\left[(s / \hbar)^{2}\right]$. However, when the contribution of the other half is added, the $O\left[(s / \hbar)^{2}\right]$ correction cancels out and Eq. (18) is obtained (see Appendix D).

We emphasize that the SRT and its implications (18) and (19) are valid for transients and for any initial state-not just for steady-state operation. In Fig. 6(a), we show the cumulative work as a function of time for a four-stroke engine and a continuous engine. The vertical lines indicate a complete cycle of the four-stroke engine. In addition to the parameter common to all examples specified before, we used $\epsilon=\gamma_{c}=\gamma_{h}=10^{-4}$, and the equivalence of work at the vertical lines is apparent. In Fig. 6(b), the field and thermal coupling were increased to $\epsilon=\gamma_{c}=\gamma_{h}=$ $5 \times 10^{-3}$. Now the engines perform differently, even at the end of each cycle. This example is a somewhat extreme situation where the system changes quite rapidly (consequence of the initial state we chose). In other cases, such as steady-state operation, the equivalence can be observed for much larger action values. (a)

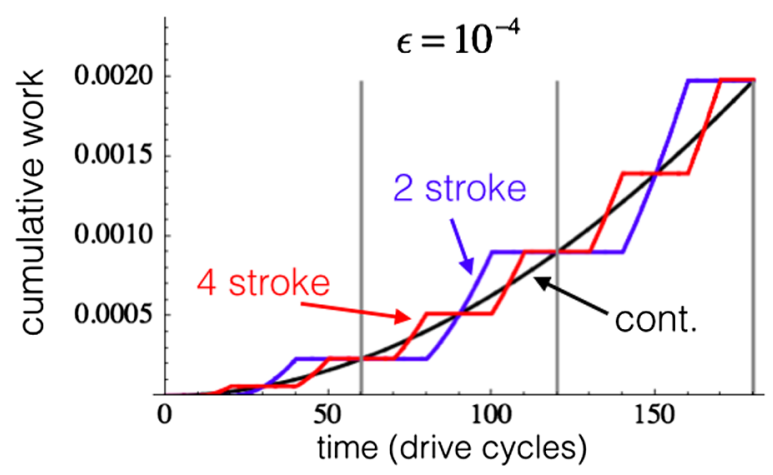

(b)

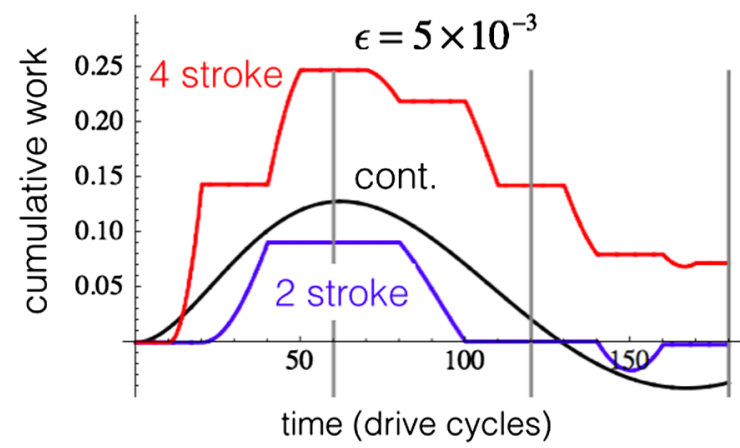

FIG. 6. (a) The equivalence of heat engine types in transient evolution when the engine action is small compared to $\hbar$. (a) The cumulative power transferred to the work repository is plotted as a function of time. All engines start in the excited state $|4\rangle$, which is very far from the steady state of the system. At complete engine cycles (vertical lines), the power in all engines is the same. (b) Once the action is increased (here, the field $\epsilon$ was increased), the equivalence no longer holds.

The splitting used in Eq. (13) was based on first splitting $\mathcal{L}_{c}$ and then $\mathcal{H}_{w}$. Other engines can be obtained by different splitting of $\tilde{\mathcal{K}}^{\text {cont }}$. For example, consider the two-stroke engine obtained by splitting $\mathcal{L}_{c}+\mathcal{L}_{h}$ :

$$
\begin{aligned}
\tilde{\mathcal{K}}^{\text {two stroke }}= & e^{-i \frac{3}{2}\left(\mathcal{L}_{c}+\mathcal{L}_{h}\right)\left(\tau_{\text {cyc }} / 3\right)} e^{-i\left(\frac{3}{2} \mathcal{H}_{w}\right)\left(\tau_{\mathrm{cyc}} / 3\right)} \\
& \times e^{-i \frac{3}{2}\left(\mathcal{L}_{c}+\mathcal{L}_{h}\right)\left(\tau_{\mathrm{cyc}} / 3\right)} .
\end{aligned}
$$

Note that in the two-stroke engine, the thermal coupling has to be $\frac{3}{2}$ stronger compared to the continuous case in order to provide the same action. Using the SRT, we obtain the complete equivalence relations of the three main engine types:

$$
\begin{aligned}
& W^{\text {two stroke }} \cong W^{\text {four stroke }} \cong W^{\text {cont }}, \\
& Q_{c(h)}^{\text {two stroke }} \cong Q_{c(h)}^{\text {four stroke }} \cong Q_{c(h)}^{\text {cont }}, \\
& \tilde{\mathcal{K}}^{\text {two stroke }} \cong \tilde{\mathcal{K}}^{\text {four stroke }} \cong \tilde{\mathcal{K}}^{\text {cont }} .
\end{aligned}
$$

Note that since $\mathcal{K}=e^{-i \mathcal{H}_{0} \tau_{\text {cyc }}} \tilde{\mathcal{K}}$, the equivalence of the evolution operators holds also in the original frame, not just in the interaction frame. Another type of engine exists when the interaction with the work repository is carried out by two physically distinct couplings. This happens 
naturally if $E_{4}-E_{3} \neq E_{2}-E_{1}$ so that two different driving lasers have to be used and the Hamiltonian is $H_{0}+\cos \left[\left(E_{2}-E_{1}\right) t\right] H_{w 1}+\cos \left[\left(E_{4}-E_{3}\right) t\right] H_{w 2}$. In such cases, one can make the splitting shown in Fig. 5(d). In this numerical example, we used $H_{w 1}=\epsilon(t)|1\rangle\langle 2|+$ H.c. and $H_{w 2}=\epsilon(t)|3\rangle\langle 4|+$ H.c. Since there are two different work strokes in addition to the thermal stroke, this engine constitutes a four-stroke engine.

\section{Power and energy flow balance}

The average power and heat flow in the equivalence regime are independent of the cycle time:

$$
\begin{gathered}
P_{W}=\frac{W}{\tau_{\mathrm{cyc}}}=\left\langle H_{0}\left|\frac{1}{2 \hbar} \mathcal{H}_{w}\right| \tilde{\rho}_{s}\right\rangle, \\
J_{c(h)}=\frac{Q_{c(h)}}{\tau_{\mathrm{cyc}}}=\left\langle H_{0}\left|\frac{1}{\hbar} \mathcal{L}_{c(h)}\right| \tilde{\rho}_{s}\right\rangle .
\end{gathered}
$$

Using the steady-state definition (15), one obtains the steady-state energy balance equation:

$$
P_{w}+J_{c}+J_{h}=0 .
$$

Equation (26) does not necessarily hold if the system is not in a steady state, as energy may be temporarily stored in the baths or in the work repository.

Figure 7 shows the power in a steady state as a function of the action. The action is increased by increasing the time duration of each stroke (see top illustration in Fig. 7). The field and the thermal coupling are $\epsilon=\gamma_{h}=\gamma_{c}=5 \times 10^{-4}$. The coupling strengths to the bath and work repository are not changed. When the engine action is large compared to $\hbar$, the engines behave very differently [Fig. 7(a)]. On the other hand, in the equivalence regime, where $s$ is small with respect to $\hbar$, the power of all engines types converges to the same value. In the equivalence regime, the power rises quadratically with the action since the correction to the power is $s^{3} / \tau_{\text {cyc }} \propto \tau_{\text {cyc }}^{2}$. This power plateau in the equivalence regime is a manifestation of quantum interference effects (coherence in the density matrix), as will be further discussed in the next section.

The behavior of different engines for large action with respect to $\hbar$ is very rich and strongly depends on the ratio between the field and the bath coupling strength.

Finally, we comment that the same formalism and results can be extended for the case in which the drive is slightly detuned from the gap.

\section{Lasing condition via the equivalence to a two-stroke engine}

Laser medium can be thought of as a continuous engine where the power output is light amplification. It is well known that lasing requires population inversion. Scovil et al. [83] were the first to show the relation between the population inversion lasing condition and the Carnot efficiency.

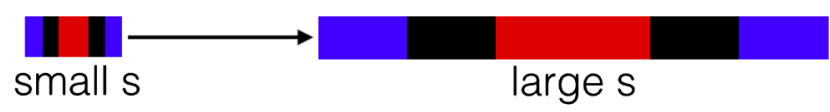

(a)

(b)

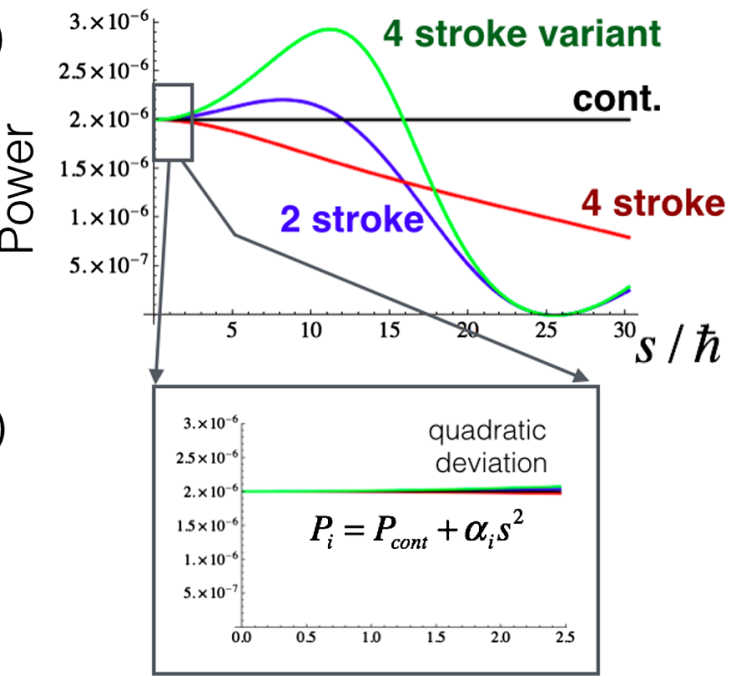

FIG. 7. Power as a function of action for various engine types in a steady state. The four-stroke variant (green line) is described in Fig. 5(d). The action is increased by increasing the stroke duration (top illustration). (a) For large action with respect to $\hbar$, the engines significantly differ in performance. In this example, all engines have the same efficiency, but they extract different amounts of heat from the hot bath. (b) In the equivalence regime where the action is small, all engine types exhibit the same power and also the same heat flows. The condition $s<\hbar / 2$ that follows from the Strang decomposition agrees with the observed regime of equivalence. The time-symmetric structure of the engines causes the deviation from equivalence to be quadratic in the action.

Using the equivalence principle presented here, the most general form of the lasing condition can be obtained without any reference to light-matter interaction.

Let us start by decomposing the continuous engine into an equivalent two-stroke engine. For simplicity, it is assumed that the hot and cold manifolds have some overlap so that, in the absence of the driving field, this bath leads the system to a unique steady state $\rho_{0}$. If the driving field is tiny with respect to the thermalization rates, then the system will be very close to $\rho_{0}$ in the steady state.

To see when $\rho_{0}$ can be used for work extraction, we need to discuss passive states. A passive state is a state that is diagonal in the energy basis, and with populations that decrease monotonically with energy [99]. The energy of a passive state cannot be decreased (or work cannot be extracted from the system) by applying some unitary transformation (the Hamiltonian after the transformation is the same as it was before the transformation) [70,99]. Thus, if $\rho_{0}$ is passive, work cannot be extracted from the device, regardless of the details of the driving field (as long as it is weak and the equivalence holds).

A combination of thermal baths will lead to an energy diagonal $\rho_{0}$. Consequently, to enable work extraction, 
passivity must be broken by population inversion. Therefore, we obtain the standard population inversion condition. Note that the derivation does not require an Einstein rate equation or any information on the processes of emission and absorption of photons.

Furthermore, it now becomes clear that if "coherent baths" are used [66] so that $\rho_{0}$ is no longer diagonal in the energy basis (and therefore no longer passive), it is possible to extract work even without population inversion.

In conclusion, using the equivalence principle, it is possible to import known results from work extraction in stroke schemes to continuous machines.

\section{QUANTUM-THERMODYNAMIC SIGNATURE}

Can the measurements of thermodynamics quantities reveal quantum effects in heat engines? To answer this, we first need to define the corresponding classical engine.

The term "classical engine" is rather ambiguous. There are different protocols of modifying the system so that it behaves classically. To make a fair comparison to the fully quantum engine, we look for the minimal modification that satisfies the following conditions:

(1) The dynamics of the device should be fully described using population dynamics (no coherences, no entanglement).

(2) The modification should not alter the energy levels of the system, the couplings to the baths, and the coupling to the work repository.

(3) The modification should not introduce a new source of heat or work.

To satisfy the first requirement, we introduce a dephasing operator that eliminates the coherences [100] and leads to a stochastic description of the engine. Clearly, a dephasing operator satisfies the second requirement. To satisfy the third requirement, we require "pure dephasing," a dephasing in the energy basis. The populations in the energy basis are invariant to this dephasing operation. Such a natural source of energy-basis dephasing emerges if there is some scheduling noise [101]. In other words, if there is some error in the switching time of the strokes.

Let us define a "quantum-thermodynamic signature" as a signal that is impossible to produce by the corresponding classical engine as defined above.

Our goal is to derive a threshold for power output that a stochastic engine cannot exceed but a coherent quantum engine can.

Before analyzing the effect of decoherence, it is instructive to distinguish between two different work extraction mechanisms in stroke engines.

\section{A. Coherent and stochastic work extraction mechanisms}

Let us consider the work done in the work stroke of a two-stroke engine [as in Fig. 5(c)]:

$$
W=\left\langle H_{0}\left|e^{-i(1 / 2 \hbar) \mathcal{H}_{w} \tau_{w}}\right| \tilde{\rho}\right\rangle-\left\langle H_{0} \mid \tilde{\rho}\right\rangle,
$$

where $\tau_{w}$ is the duration of the work stroke. Writing the state as a sum of population and coherences $|\tilde{\rho}\rangle=\left|\tilde{\rho}_{\text {pop }}\right\rangle+\left|\tilde{\rho}_{\text {coh }}\right\rangle$, we get

$$
\begin{aligned}
W= & \left\langle H_{0}\left|\sum_{n=1} \frac{\left(-i \frac{1}{2 \hbar} \mathcal{H}_{w} \tau_{w}\right)^{2 n-1}}{(2 n-1) !}\right| \tilde{\rho}_{\mathrm{coh}}\right\rangle \\
& +\left\langle H_{0}\left|\sum_{n=1} \frac{\left(-i \frac{1}{2 \hbar} \mathcal{H}_{w} \tau_{w}\right)^{2 n}}{(2 n) !}\right| \tilde{\rho}_{\mathrm{pop}}\right\rangle .
\end{aligned}
$$

This result follows from the generic structure of Hamiltonians in Liouville space. Any $\mathcal{H}$ that originates from a Hermitian Hamiltonian in Hilbert space (in contrast to Lindblad operators as a source) has the structure shown in Fig. 8(b) (see Appendix A for Liouville space derivation of this property). In other words, it connects only populations to coherences and vice versa, but it cannot connect populations to populations directly [102]. In addition, since $\left\langle H_{0}\right|$ acts as a projection on population space, one gets that odd powers of $\mathcal{H}_{w}$ can only operate on coherences and even powers can only operate on populations. Thus, the power can be extracted using two different mechanisms: a coherent mechanism that operates on coherences and a stochastic mechanism that operates on populations.

The effects of the "stochastic" terms $\sum_{n=1}\left(-i \frac{1}{2 \hbar} \mathcal{H}_{w} \tau_{w}\right)^{2 n} /(2 n)$ ! on the populations are equivalently described by a single doubly stochastic operator. If there are no coherences (next section), this leads to a simple interpretation in terms of full swap events that take place with some probability.

(a)
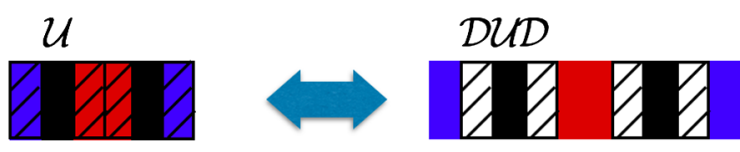

(b)

$$
|\rho\rangle=\left(\begin{array}{c}
\text { pop } \\
\hdashline \text { coh }
\end{array}\right) \quad \mathcal{H}=\mathcal{H}^{\dagger}=\left(\begin{array}{c:c}
0 & h \\
\hdashline h^{\dagger} & 0 \\
\text { pop } & \text { coh }
\end{array}\right) \text { pop }
$$

FIG. 8. Panel (a), left side: Dephasing operations (slanted line, operator $\mathcal{D}$ ) commute with thermal baths so the dephased engine in the left side of (a) is equivalent to the one on the right. In the new engine, the unitary evolution is replace by $\mathcal{D} \mathcal{U D}$. If $\mathcal{D}$ eliminates all coherences, the effect of $\mathcal{D U D}$ on the populations can always be written as a doubly stochastic operator. (b) Any Hermitian Hamiltonian in Liouville space has the structure shown in (b). Thus, first-order changes in populations critically depend on the existence of coherence. 
Continuous engines, on the other hand, have only a coherent work extraction mechanism. This can be seen from the expression for their work output,

$$
P^{\mathrm{cont}}=\left\langle H_{0}\left|\frac{1}{2 \hbar} \mathcal{H}_{w}\right| \tilde{\rho}\right\rangle=\left\langle H_{0}\left|\frac{1}{2 \hbar} \mathcal{H}_{w}\right| \tilde{\rho}_{\mathrm{coh}}\right\rangle,
$$

where again we used the population projection property of $\left\langle H_{0}\right|$ and the structure of $\mathcal{H}_{w}$ [Fig. 8(b)]. We conclude that in contrast to stroke engines, continuous engines have no stochastic work extraction mechanism. This difference stems from the fact that in continuous engines, the steady state is stationary. Consequently, there are no higher-order terms that can give rise to a population-population stochastic work extraction mechanism. This is a fundamental difference between stroke engines and continuous engines. This effect is pronounced outside the equivalence regime where the stochastic terms become important (see Sec. V).

\section{B. Engines subjected to pure dephasing}

Consider the engine shown in Fig. 8(a). The slanted lines on the baths indicate that there is an additional dephasing mechanism that takes place in parallel to the thermalization [103]. Let us denote the evolution operator of the pure dephasing by $\mathcal{D}$. In principle, to analyze the deviation from the coherent quantum engine, first the steady state has to be solved and then work and heat can be compared. Even for simple systems, this is a difficult task. Hence, we shall take a different approach and derive an upper bound for the power of stochastic engines. It is important that the bound contains only quantities that are unaffected by the level of coherence in the system. For example, the dipole expectation value, does contain information on the coherence. We construct a bound in terms of the parameters of the system (e.g., the energy levels, coupling strengths, etc.), which is independent of the state of the system. In the pure dephasing stage, the energy does not change. Hence, the total energy change in the $\mathcal{D U D}$ stage is associated with work.

Let $\mathcal{D}_{\text {comp }}=\mid$ pop $\rangle\langle$ pop $|$ be a projection operator on the population space. This operator generates a complete dephasing that eliminates all coherences. In such a case, the leading order in the work expression becomes

$$
\begin{aligned}
W & =\left\langle H_{0}\left|\mathcal{D}_{\text {comp }} e^{-i(1 / 2 \hbar) \mathcal{H}_{w} \tau_{w}} \mathcal{D}_{\text {comp }}\right| \tilde{\rho}\right\rangle \\
& =\frac{\tau_{w}^{2}}{8 \hbar^{2}}\left\langle H_{0}\left|\mathcal{H}_{w}^{2}\right| \tilde{\rho}_{\mathrm{pop}}\right\rangle+O\left[(s / \hbar)^{4}\right]
\end{aligned}
$$

where we used $\left\langle H_{0}\right| \mathcal{D}=\left\langle H_{0}\right|$ and $\mathcal{D}_{\text {comp }}|\tilde{\rho}\rangle=\left|\tilde{\rho}_{\text {pop }}\right\rangle$. Since $\mathcal{D}_{\text {comp }}$ eliminates coherences, $W$ does not contain a linear term in time. Next, by using the following relation, $\left\langle H_{0}|B| \rho\right\rangle \leq \sqrt{\left\langle H_{0} \mid H_{0}\right\rangle\langle\rho \mid \rho\rangle}\|B\|, \sqrt{\left\langle H_{0} \mid H_{0}\right\rangle}=\sqrt{\operatorname{tr}\left(H_{0}^{2}\right)}$, we find that for $s \ll \hbar$ the power of a stochastic engine satisfies

$$
\begin{aligned}
P_{\text {stoch }} & \leq \frac{z}{8 \hbar^{2}} \sqrt{\operatorname{tr}\left(H_{0}^{2}\right)-\operatorname{tr}\left(H_{0}\right)^{2}} \Delta_{w}^{2} d^{2} \tau_{\text {cyc }}, \\
z & =1 \text { two-stroke } \\
z & =1 / 2 \text { four-stroke }
\end{aligned}
$$

where $\Delta_{w}$ is the gap of the interaction Hamiltonian (maximal eigenvalue minus minimal eigenvalue of $H_{w}$ ), and $d$ is the duty cycle - the fraction of time dedicated to work extraction ( $d=\tau_{w} / \tau_{\text {cyc }}$, e.g., $d=1 / 3$ in all the examples in this paper). We also used the fact that $\left\langle\rho_{\text {pop }} \mid \rho_{\text {pop }}\right\rangle$ is always smaller than the purity $\langle\rho \mid \rho\rangle$ and therefore smaller than 1 . Note that, as we required, this bound is state independent, and the right-hand side of Eq. (31) contains no information on the coherences in the system. Thus, we conclude that for power measurements,

$P>P_{\text {stoch }} \Rightarrow$ quantum-thermodynamic signature.

As shown earlier, in coherent quantum engines (in the equivalence regime), the work scales linearly with $\tau_{\text {cyc }}$ [see Eqs. (16) and (18)], and therefore, the power is constant as a function of $\tau_{\text {cyc }}$. When there are no coherences, the power scales linearly with $\tau_{\text {cyc }}$.

Numerical results of power as a function of cycle time are shown in Fig. 9. The power is not plotted as a function of action as before because, at the same cycle time, the coherent engine and the dephased engine have different actions. The coupling parameters are as in Fig. 7. The action of the dephased engine is

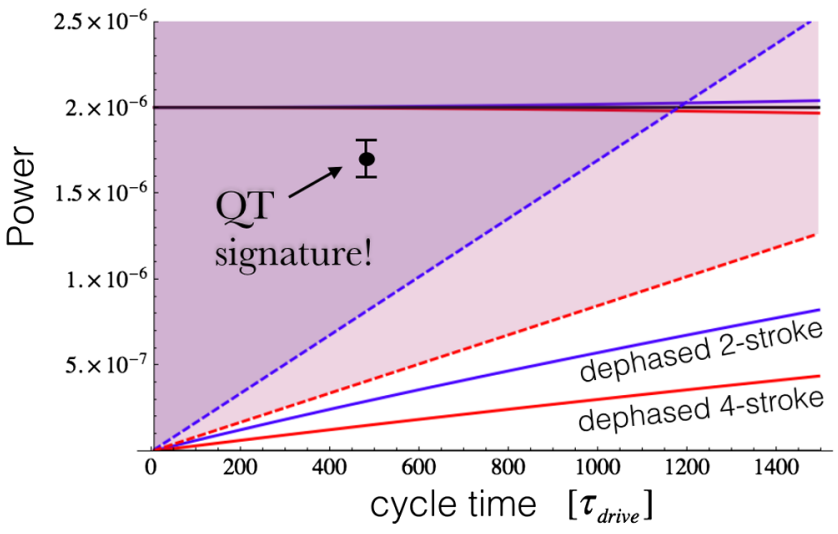

FIG. 9. The power output of the three types of engines (twostroke blue, four-stroke red, continuous black) with and without dephasing [top horizontal solid lines are without dephasingsame as in Fig. 7(b)]. The power of the continuous dephased engine is zero. The dashed lines show the stochastic upper bounds on the power of two-stroke (dashed blue line) and four-stroke (dashed red line) engines. Any power measurement in the shaded area of each engine indicates the presence of quantum interference in the engine. This plot also demonstrates that for short cycle times (low action), coherent engines produce much more power compared to stochastic dephased engines. 


$$
s_{\text {deph }}=\left(\left\|\mathcal{L}_{c}\right\|+\left\|\mathcal{L}_{h}\right\|+\left\|\frac{1}{2} \mathcal{H}_{w}\right\|+\left\|\mathcal{L}_{\text {dephasing }}\right\|\right) \tau_{\text {cyc }}
$$

If the dephasing is significant, the action is large and equivalence cannot be observed. In other words, a fully stochastic engine in a quantum system has a large action and cannot satisfy $s \ll \hbar$.

The stochastic power bounds for a two-stroke engine (dashed blue line) and for a four-stroke engine (dashed red line) define a power regime (shaded areas) that is inaccessible to fully stochastic engines. Thus, any power measurement in this regime unequivocally indicates the presence of quantum coherences in the engine.

In practice, the dephasing time may be very small but different from zero. When the cycle time is large compared to the dephasing time, the system behaves as if there is complete dephasing. If, however, the cycle time is small with respect to the decoherence time (close to the origin of Fig. 9), the power will form a plateau of finite power instead of reducing to zero.

Note that to measure power, the measurement is carried out on the work repository and not on the engine. Furthermore, the engine must operate for many cycles to reduce fluctuations in the accumulated work. To calculate the average power, the accumulated work is divided by the total operation time and compared to the stochastic power threshold (31).

Also, note that a complete dephasing would have resulted in zero power output for the continuous engine (29).

In summary, the quantum-thermodynamics signature in stroke engines can be observed in the weak action limit.

\section{OVER-THERMALIZATION EFFECT IN COHERENT QUANTUM HEAT ENGINE}

In all the numerical examples studied so far, the unitary action and the thermal action were roughly comparable for reasons that will soon become clear. In this section, we study some generic features that take place when the thermal action takes over.

Let us now consider the case where the unitary contribution to the action $\left\|\mathcal{H}_{\omega}\right\| \tau$ is small with respect to $\hbar$. All the time intervals are fixed, but we can control the thermalization rate $\gamma$ (for simplicity, we assume it is the same value for both baths). Common sense suggests that increasing $\gamma$ should increase the power output. At some stage, this increase will stop since the system will already reach thermal equilibrium with the bath (or baths in twostroke engines). Yet, Fig. 10 shows that there is a very distinctive peak where an optimal coupling takes place. In other words, in some cases, less thermalization leads to more power. We call this effect over-thermalization. This effect is generic and not unique to the specific model used

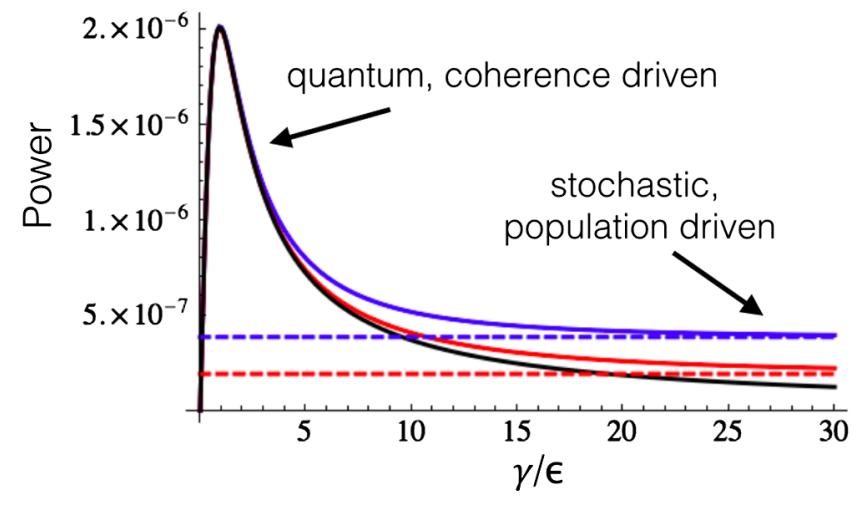

FIG. 10. The over-thermalization effect is the decrease of power when the thermalization rate is increased. Over-thermalization degrades the coherent work extraction mechanism without affecting the stochastic work extraction mechanism. When the coherent mechanism gets weak enough, the power is dominated by the stochastic power extraction mechanisms and power saturation is observed (dashed lines). The continuous engine has no stochastic work extraction mechanism, and therefore, it decays to zero without reaching saturation.

in the numerical simulations. The parameters used for the plot are $\epsilon=\gamma_{c}=\gamma_{h}=2 \times 10^{-4}$, and the number of drives cycles per engine cycle is $m=600$.

The peak and the saturation are a consequence of the interplay between the two different work extraction mechanisms (see Sec. IVA). For low $\gamma$, the coherences in the system are significant, and the leading term in the power is $\left\langle H_{0}\left|-i(1 / 2 \hbar) \mathcal{H}_{w}\right| \tilde{\rho}_{\text {coh }}\right\rangle d$ (where $d$ is the duty cycle). In principle, all Lindblad thermalization processes are associated with some level of decoherence. This decoherence generates an exponential decay of $\left|\tilde{\rho}_{\text {coh }}\right\rangle$ that explains the decay on the right-hand side of the peak. At a certain stage, the linear term becomes so small that the stochastic secondorder term $-\left(1 / 8 \hbar^{2}\right)\left\langle H_{0}\left|\mathcal{H}_{w}^{2}\right| \tilde{\rho}_{\text {pop }}\right\rangle d^{2} \tau_{\text {cyc }}$ dominates the power. $\left|\tilde{\rho}_{\text {pop }}\right\rangle$ eventually saturates for large $\gamma$, and therefore, the stochastic second-order term leads to a power saturation. Interestingly, in the example shown in Fig. 10, we observe that the peak is obtained when $\gamma$ and $\epsilon$ are roughly equal. Of course, what really matters is the thermal action with respect to unitary action and not just the values of the parameters $\gamma$ and $\epsilon$. We point out that this effect for a continuous engine can be seen in Fig. 3 of Ref. [20] and in Fig. 11 of Ref. [104]. In the present work, the mechanism that generates this general effect has been clarified.

If thermalization occurs faster, the thermal stroke can be shortened, and this increases the power. However, this effect is small with respect to the exponential decay of the coherences. We conclude that even without additional dephasing as in the previous section, excessive thermal coupling turns the engine into a stochastic machine. For small unitary action, this effect severely degrades the power output. The arguments presented here are valid for any small-action coherent quantum engine. 


\section{CONCLUDING REMARKS}

We identified coherent and stochastic work extraction mechanisms in quantum heat engines. While stroke engines have both mechanisms, continuous engines only have the coherent mechanism. We introduced the "norm action" of the engine using Liouville space and showed that when this action is small compared to $\hbar$, all three engine types are equivalent. This equivalence emerges because, for small actions, only the coherent mechanism is important. Despite the equivalence, before the engine cycle is completed, the state of the different engine type differs by $O\left[(s / \hbar)^{1}\right]$. This also holds true for work and heat. Remarkably, at the end of each engine cycle, a much more accurate $O\left[(s / \hbar)^{3}\right]$ equivalence emerges. Furthermore, the equivalence also holds for transient dynamics, even when the initial state is very far from the steady state of the engine. It was shown that, for small actions, the coherent work extraction is considerably stronger than the stochastic work extraction mechanism. This enabled us to derive a power bound for stochastic engines that constitutes a quantum-thermodynamics signature. Any power measurement that exceeds this bound indicates the presence of quantum coherence and the operation of the coherent work extraction mechanism.

Experimental schemes where the work is extracted by changing the energy levels (e.g., Refs. [53-55]) correspond to a full swap in the multilevel embedding framework. Consequently, such setups have an inherently large action, and they are not suited for demonstrating the effects presented here. In contrast, the scheme in Ref. [105] seems highly suitable. There, the unitary operation that makes a swap between superconducting qubits is generated by creating a magnetic flux through a superconducting ring. In the original paper, the authors use a flux that generates a full swap. However, by using weaker magnetic fields, the unitary operation will become a partial swap, and it should be possible to attain the small action regime where the equivalence can be observed. In addition, NV centers in diamonds also have the potential for exploring heat engine equivalence in the quantum regime.

The present derivation makes no assumption on the direction of heat flows and the sign of work. Thus, our results are equally applicable to refrigerators and heaters.

It is interesting to try and apply these concepts of equivalence and quantum-thermodynamic signatures to more general scenarios: non-Markovian baths, engines with a nonsymmetric unit cell, and engines with quantum correlation between different particles (entanglement and quantum discord). We conjecture that in multiple particle engines, entanglement will play a similar role to that of coherence in single-particle engines.

\section{ACKNOWLEDGMENTS}

This work was support by the Israeli Science Foundation. Part of this work was supported by the
COST Action MP1209 "Thermodynamics in the quantum regime."

\section{APPENDIX A: LIOUVILLE SPACE FORMULATION OF QUANTUM DYNAMICS}

Quantum dynamics is traditionally described in Hilbert space. However, it is convenient, in particular, for open quantum systems, to introduce an extended space where density operators are vectors and time evolution is generated by a Schrödinger-like equation. This space is usually referred to as Liouville space [89]. We denote the "density vector" by $|\rho\rangle \in \mathbb{C}^{1 \times N^{2}}$. It is obtained by reshaping the density matrix $\rho$ into a larger single vector with index $\alpha \in\left\{1,2, \ldots N^{2}\right\}$. The one-to-one mapping of the two matrix indices into a single vector index $\{i, j\} \rightarrow \alpha$ is arbitrary but has to be used consistently. The vector $|\rho\rangle$ is not normalized to unity, in general. Its norm is equal to the purity, $\mathcal{P}=\operatorname{tr}\left(\rho^{2}\right)=\langle\rho \mid \rho\rangle$, where $\langle\rho|=| \rho\rangle^{\dagger}$ as usual. The equation of motion of the density vector in Liouville space follows from $d_{t} \rho_{\alpha}=\sum_{\beta} \rho_{\beta} \partial\left(d_{t} \rho_{\alpha}\right) / \partial \rho_{\beta}$. Using this equation, one can verify that the dynamics of the density vector $|r\rangle$ is governed by a Schrödinger-like equation in the new space,

$$
i \partial_{t}|\rho\rangle=\mathcal{H}|\rho\rangle,
$$

where the super-Hamiltonian $\mathcal{H} \in \mathbb{C}^{N^{2} \times N^{2}}$ is given by

$$
\mathcal{H}_{\alpha \beta}=i \frac{\partial\left(d_{t} \rho_{\alpha}\right)}{\partial \rho_{\beta}} .
$$

A particularly useful index mapping is described in Ref. [106] and in Ref. [90]. In this mapping, the Liouville index of $|\rho\rangle$ is related to the original row and column index of $\rho$ via $\alpha=\mathrm{col}+N($ row -1$)$. For this form, $\mathcal{H}$ can be compactly written in term of the original $H$ and A:

$$
\begin{aligned}
\mathcal{H}= & -i\left(H \otimes I-I \otimes H^{t}\right) \\
& +i \sum_{k}\left[\left(A_{k} \otimes A_{k}^{*}\right)-\frac{1}{2} I \otimes\left(A_{k}^{\dagger} A_{k}\right)^{t}-\frac{1}{2} A_{k}^{\dagger} A_{k} \otimes I\right],
\end{aligned}
$$

where the superscript $t$ stands for transposition and $*$ for complex conjugation. $\mathcal{H}=\mathcal{H}^{H}+\mathcal{L}$ is non-Hermitian for open quantum systems. $\mathcal{H}^{H}$ originates from the Hilbert space Hamiltonian $H$, and $\mathcal{L}$ from the Lindblad terms. $\mathcal{H}^{H}$ is always Hermitian. The skew-Hermitian part $\left(\mathcal{L}-\mathcal{L}^{\dagger}\right) / 2$ is responsible for purity changes. Yet, in Liouville space, the Lindblad operators $A_{k}$ in Eq. (1) may also generate a Hermitian term $\left(\mathcal{L}+\mathcal{L}^{\dagger}\right) / 2$. Though Hermitian in Liouville space, this term cannot be associated with a Hamiltonian in Hilbert space. If $\mathcal{L}=0, \mathcal{K}$ is unitary. It is important to note that not all eigenvectors of $\mathcal{H}$ in Liouville 
space can be populated exclusively. This is due to the fact that only positive $\rho$ with unit trace are legitimate density matrices. The states that can be populated exclusively describe steady states, while others correspond to transient changes. We remind the reader that, in this paper, we use calligraphic letters to describe operators in Liouville space and ordinary letters for operators in Hilbert space. For states, however, $|A\rangle$ will denote a vector in Liouville space formed from $A_{N \times N}$ by "vec-ing" $A$ into a column in the same procedure $\rho$ is converted into $|\rho\rangle$.

\section{Useful relations in Liouville space}

In Liouville space, the standard inner product of two operators in Hilbert space $\operatorname{tr} A^{\dagger} B$ reads

$$
\operatorname{tr} A^{\dagger} B=\langle A \mid B\rangle .
$$

In particular, the purity $\mathcal{P}=\langle r \mid r\rangle$ is just the square of the distance from the origin in Liouville space.

A useful relation for $\mathcal{H}^{H}$ is

$$
\mathcal{H}^{H}|H\rangle=\langle H| \mathcal{H}^{H}=0 .
$$

The proof is as follows:

$$
\mathcal{H}_{i j, m n}^{H}=H_{i m} \delta_{j n}-H_{n j} \delta_{i m} .
$$

Therefore, using Eq. (A5) we get

$$
\mathcal{H}^{H}|H\rangle=\sum_{\beta} \mathcal{H}_{\alpha \beta}^{H} H_{\beta}=\sum_{m n} \mathcal{H}_{i j m n}^{H} H_{m n}=[H, H]=0 .
$$

This property is highly useful. We stress that Eq. (A4) is a property of Hermitian operators in Hilbert space, where both $H$ and $\mathcal{H}$ are well defined. A general Hermitian operator in Liouville space may not have a corresponding $H$ in Hilbert space.

Another property that immediately follows from Eq. (A5) is

$$
\mathcal{H}_{i i, k k}^{H}=0 .
$$

This corresponds to a well-known property of unitary operation. If the system starts from a diagonal density matrix, then for short times, the evolution generated by $\mathcal{H}^{H}$, $e^{-i \mathcal{H}^{H} d t}=I-i \mathcal{H}^{H} d t+O\left(d t^{2}\right)$ does not change the population in the leading order.

\section{Expectation values and their time evolution in Liouville space}

The expectation value of an operator in Hilbert space is $\langle A\rangle=\operatorname{tr}(\rho A)$. Since $\rho$ is Hermitian, the expectation value is equal to the inner product of $A$ and $\rho$, and therefore,

$$
\langle A\rangle=\operatorname{tr}(\rho A)=\langle\rho \mid A\rangle .
$$

The dynamics of $\langle A\rangle$ under the Lindblad evolution operator is

$$
\frac{d}{d t}\langle A\rangle=-i\langle A|\mathcal{H}| \rho\rangle+\left\langle\rho \mid \frac{d}{d t} A\right\rangle .
$$

Note that in Liouville space there is no commutator term since $\mathcal{H}$ operates on $|\rho\rangle$ just from the left. If the total Hamiltonian is Hermitian and time independent, the conservation of energy follows immediately from applying Eqs. (A8) and (A4) for $A=H$.

\section{APPENDIX B: GEOMETRIC MEANING OF THE NORM ACTION}

This appendix establishes the relation between the norm action and the path length in Liouville space. The action constitutes an upper bound on the length of the path over one cycle. The infinitesimal path $d l$ between two states $|\rho(t+d t)\rangle$ and $|\rho(t)\rangle$ in Liouville space is given by

$$
\begin{aligned}
d l^{2} & =\||\rho(t+d t)\rangle-|\rho(t)\rangle \|_{2}^{2} \\
& =\left\langle\rho(t)\left|\mathcal{H}^{\dagger} \mathcal{H}\right| \rho(t)\right\rangle d t^{2} / \hbar^{2}+O\left(d t^{3}\right),
\end{aligned}
$$

where $\quad \||\rho(t+d t)\rangle-|\rho(t)\rangle \|_{2}^{2}=\operatorname{tr}\left([\rho(t+d t)-\rho(t)]^{2}\right)$. Consequently, the path in Liouville space is given by

$$
L=\int_{0}^{\tau_{\mathrm{cyc}}}\left(\frac{d l}{d t}\right) d t \leq \frac{1}{\hbar} \int_{0}^{\tau_{\mathrm{cyc}}}\|\mathcal{H}\|\langle\rho \mid \rho\rangle d t
$$

where we have used the property of the spectral norm $\left\langle\rho(t)\left|\mathcal{H}^{\dagger} \mathcal{H}\right| \rho(t)\right\rangle /\langle\rho \mid \rho\rangle \leq\|\mathcal{H}\|_{\text {sp. }}^{2}$. Since the purity $\langle\rho \mid \rho\rangle$ is always smaller than 1 ,

$$
L \leq \frac{1}{\hbar} \int_{0}^{\tau_{\mathrm{cyc}}}\|\mathcal{H}\| d t \equiv s / \hbar
$$

Thus, the path length per cycle in Liouville space is bounded by the action. For previous uses of the norm action to quantify quantum dynamics, see Refs. [107-110]. This is also true for times shorter than the cycle time $\tau_{\text {cyc }}$,

$$
L(\tau)=\int_{0}^{\tau}\left(\frac{d l}{d t}\right) d t \leq s / \hbar .
$$

The triangle inequality implies $\||\rho(\tau)\rangle-|\rho(0)\rangle \|_{2} \leq L(\tau)$; therefore,

$$
\max \left(\||\rho(\tau)\rangle-|\rho(0)\rangle \|_{2}\right) \leq s / \hbar .
$$

Hence, the action limits the maximal state change during the cycle. For example, if the action is $10^{-3} \hbar$, the state will change by $10^{-3}$ at the most. 


\section{APPENDIX C: STRANG DECOMPOSITION VALIDITY}

Let $\mathcal{K}$ be an operator generated by two noncommuting operators $\mathcal{A}$ and $\mathcal{B}$ :

$$
\mathcal{K}=e^{(\mathcal{A}+\mathcal{B}) d \lambda},
$$

where we use $d \lambda=d t / \hbar$ for brevity. The splitted operator is

$$
\mathcal{K}_{s}=e^{\frac{1}{2} \mathcal{A} d \lambda} e^{\mathcal{B} d \lambda} e^{\frac{1}{2} \mathcal{A} d \lambda} .
$$

Our goal is to quantify the difference between $\mathcal{K}$ and $\mathcal{K}_{s}$, $\left\|\mathcal{K}_{s}-\mathcal{K}\right\|$, where $\|\cdot\|$ stands for the spectral norm. In principle, other submultiplicative matrix norms can be used (such as the Hilbert-Schmidt norm). However, the spectral norm more accurately captures aspects of quantum dynamics $[108-111] . \mathcal{K}$ can be expanded as

$$
\mathcal{K}=\sum \frac{(\mathcal{A}+\mathcal{B})^{n} d \lambda^{n}}{n !}
$$

$\mathcal{K}_{s}$, on the other hand, is

$$
\begin{aligned}
\mathcal{K}_{s} & =\sum_{k, l, m=0}^{\infty} \frac{(\mathcal{A} / 2)^{k} d \lambda^{k}}{k !} \frac{\mathcal{B}^{l} d \lambda^{l}}{l !} \frac{(\mathcal{A} / 2)^{m} d \lambda^{m}}{m !} \\
& =\sum_{n=0}^{\infty} \sum_{l=0}^{n} \sum_{k=0}^{n-l} \frac{(\mathcal{A} / 2)^{k}}{k !} \frac{\mathcal{B}^{l}}{l !} \frac{(\mathcal{A} / 2)^{n-l-k}}{(n-l-k) !} d \lambda^{n} .
\end{aligned}
$$

Because of the symmetric splitting, the terms up to $n=2$ (including $n=2$ ) are identical for both operators. Therefore, the difference can be written as

$$
\begin{aligned}
\left\|\mathcal{K}_{s}-\mathcal{K}\right\|= & \| \sum_{n=3}^{\infty} \sum_{l=0}^{n} \sum_{k=0}^{n-l} \frac{(\mathcal{A} / 2)^{k}}{k !} \frac{\mathcal{B}^{l}}{l !} \frac{(\mathcal{A} / 2)^{n-l-k}}{(n-l-k) !} d \lambda^{n} \\
& -\sum_{n=3} \frac{(\mathcal{A}+\mathcal{B})^{n} d \lambda^{n}}{n !} \| .
\end{aligned}
$$

Next, we apply the triangle inequality and the submultiplicativity property to get

$$
\begin{aligned}
\left\|\mathcal{K}_{s}-\mathcal{K}\right\| \leq & \| \sum_{n=3}^{\infty} \sum_{l=0}^{n} \sum_{k=0}^{n-l} \frac{\|\mathcal{A} / 2\|^{k}}{k !} \frac{\|B\|^{l}}{l !} \frac{\|\mathcal{A} / 2\|^{n-l-k}}{(n-l-k) !} d \lambda^{n} \\
& +\sum_{n=3}^{\infty} \frac{(\|\mathcal{A}\|+\|\mathcal{B}\|)^{n} d \lambda^{n}}{n !} \| .
\end{aligned}
$$

Using the binomial formula two times, one finds

$$
\begin{aligned}
& \sum_{n=3}^{\infty} \sum_{l=0}^{n} \sum_{k=0}^{n-l} \frac{\|\mathcal{A} / 2\|^{k}}{k !} \frac{\|\mathcal{B}\|^{l}}{l !} \frac{\|\mathcal{A} / 2\|^{n-l-k}}{(n-l-k) !} d \lambda^{n} \\
& \quad=\sum_{n=3}^{\infty} \frac{(\|\mathcal{A}\|+\|\mathcal{B}\|)^{n} d \lambda^{n}}{n !}
\end{aligned}
$$

and therefore,

$$
\begin{aligned}
\left\|\mathcal{K}_{s}-\mathcal{K}\right\| & \leq 2 \sum_{n=3}^{\infty} \frac{(\|\mathcal{A}\|+\|\mathcal{B}\|)^{n} d \lambda^{n}}{n !} \\
& =2 R_{2}[(\|\mathcal{A}\|+\|\mathcal{B}\|) d \lambda] .
\end{aligned}
$$

The right-hand side is the Taylor remainder of a power series of an exponential with $(\|\mathcal{A}\|+\|\mathcal{B}\|) d \lambda$ as an argument. The Taylor remainder formula for the exponent function is $R_{k}(x)=e^{\xi}\left(|x|^{k+1}\right) /(k+1)$ !, where $0 \leq \xi \leq 1$ (for now, we assume $x<1$ ). Setting $k=2$ and $\xi=1$ (worst case), we finally obtain

$$
\begin{gathered}
\left\|\mathcal{K}_{s}-\mathcal{K}\right\| \leq \frac{e}{3}[(\|A\|+\|\mathcal{B}\|) d \lambda]^{3} \leq\left(\frac{s}{\hbar}\right)^{3} \\
s=(\|A\|+\|\mathcal{B}\|) d t
\end{gathered}
$$

where we call $s$ the norm action of the evolution operator. To get an estimation where the leading non-neglected term of $\mathcal{K},(\mathcal{A}+\mathcal{B})^{2} d \lambda^{2} / 2$, is larger than the remainder, we require that

$$
(\|\mathcal{A}+\mathcal{B}\|)^{2} d \lambda^{2} / 2 \geq\left(\frac{s}{\hbar}\right)^{3}
$$

Using the triangle inequality, we get the estimated condition for the Strang decomposition:

$$
s \leq \hbar / 2 \text {. }
$$

This condition explains why it was legitimate to limit the range of $x$ to 1 in the remainder formula.

\section{APPENDIX D: SYMMETRIC REARRANGEMENT THEOREM}

The goal of this appendix is to explain why the equivalence of evolution operators leads to equivalence of work and equivalence of heat. In addition, we show why this is also valid for transients. For the equivalence of the evolution operator, we require that the super-Hamiltonian is symmetric and that the action is small:

$$
\begin{gathered}
\mathcal{H}(t)=\mathcal{H}(-t), \\
s=\int_{-\tau / 2}^{+\tau / 2}\|\mathcal{H}\| d t \ll \hbar .
\end{gathered}
$$


Let the initial state at time $t=-\tau / 2$ be

$$
\left|\tilde{\rho}_{i}\right\rangle=\mid \tilde{\rho}(-\tau / 2\rangle .
$$

This state leads to a final state at $\tau / 2$,

$$
\left|\tilde{\rho}_{f}\right\rangle=\mid \tilde{\rho}(\tau / 2\rangle .
$$

Our goal is to evaluate a symmetric expectation value difference of the form

$$
\begin{aligned}
d A_{\mathrm{tot}}= & {\left[\left\langle A\left(t_{2}\right)\right\rangle-\left\langle A\left(t_{1}\right)\right\rangle\right]+\left[\left\langle A\left(-t_{1}\right)\right\rangle-\left\langle A\left(-t_{2}\right)\right\rangle\right] } \\
= & {\left[\left\langle A \mid \tilde{\rho}\left(t_{2}\right)\right\rangle-\left\langle A \mid \tilde{\rho}\left(t_{1}\right)\right\rangle\right] } \\
& +\left[\left\langle A \mid \tilde{\rho}\left(-t_{1}\right)\right\rangle-\left\langle A \mid \tilde{\rho}\left(-t_{2}\right)\right\rangle\right], \\
t_{2}, t_{1} \geq & 0,
\end{aligned}
$$

$$
\begin{aligned}
& \left|\tilde{\rho}\left(t_{1}\right)\right\rangle=|\tilde{\rho}(0)\rangle-i \int_{0}^{t_{1}} \mathcal{H}(t) \frac{d t}{\hbar}|\tilde{\rho}(0)\rangle+O\left[\left(\frac{s}{\hbar}\right)^{2}\right] \\
& \left|\tilde{\rho}\left(-t_{1}\right)\right\rangle=|\tilde{\rho}(0)\rangle+i \int_{0}^{t_{1}} \mathcal{H}(t) \frac{d t}{\hbar}|\tilde{\rho}(0)\rangle+O\left[\left(\frac{s}{\hbar}\right)^{2}\right] .
\end{aligned}
$$

segment $\left[t_{1}, t_{2}\right]$ and its symmetric counterpart in negative time [e.g., the green areas in Fig. 11(a)]. When $A$ is equal to $H_{0}$, this difference will translate into work or heat. We start with the expansion

$$
\begin{aligned}
& {\left[\left\langle A\left(t_{2}\right)\right\rangle-\left\langle A\left(t_{1}\right)\right\rangle\right]=\left\langle A\left|\mathcal{K}_{t_{1} \rightarrow t_{2}}-I\right| \tilde{\rho}\left(t_{1}\right)\right\rangle} \\
& =\left\langle A\left|-i \mathcal{H}\left(t_{1}\right) \frac{\delta t}{\hbar}-\frac{1}{2} \mathcal{H}\left(t_{1}\right)^{2} \frac{\delta t^{2}}{\hbar^{2}}\right| \tilde{\rho}\left(t_{1}\right)\right\rangle \\
& \quad+O\left[\left(\frac{s}{\hbar}\right)^{3}\right] .
\end{aligned}
$$

For the negative side, we get

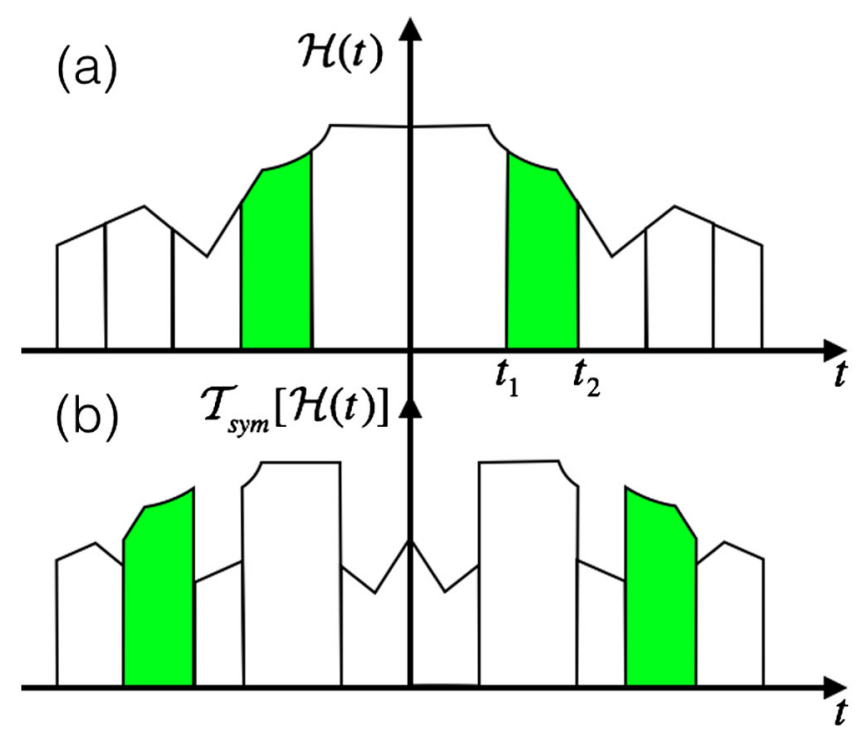

FIG. 11. The Hamiltonians in (a) and (b) are related by symmetric rearrangement of the time segments. Up to a small correction $O\left(s^{3}\right)$, the change in expectation values of an observable $A$ that takes place during the green segments is the same in both cases. This effect explains why work and heat are the same in various types of engines when $s$ is small compared to $\hbar$ (equivalence regime).

$$
\begin{aligned}
& {\left[\left\langle A\left(-t_{1}\right)\right\rangle-\left\langle A\left(-t_{2}\right)\right\rangle\right]=\left\langle A\left|I-\mathcal{K}_{-t_{1} \rightarrow-t_{2}}\right| r\left(-t_{1}\right)\right\rangle} \\
& =\left\langle A\left|-i \mathcal{H}\left(-t_{1}\right) \frac{\delta t}{\hbar}+\frac{1}{2} \mathcal{H}\left(-t_{1}\right)^{2} \frac{\delta t^{2}}{\hbar^{2}}\right| \tilde{\rho}\left(-t_{1}\right)\right\rangle \\
& \quad+O\left[\left(\frac{s}{\hbar}\right)^{3}\right] .
\end{aligned}
$$

Next, we use the fact that

When adding the two segments, the second order terms cancel out and we get

$$
\delta A_{\mathrm{tot}}=-2 i\left\langle A\left|\mathcal{H}\left(t_{1}\right)\right| \tilde{\rho}(0)\right\rangle \delta t+O\left[\left(\frac{s}{\hbar}\right)^{3}\right] .
$$

Note that the result is expressed using $|\tilde{\rho}(0)\rangle$, which is not given explicitly. To correctly relate it to $|\tilde{\rho}(-\tau / 2)\rangle$, we have to use the symmetric rearrangement properties of the evolution operator.

\section{Symmetric rearrangement}

In Fig. 11(a), there is an illustration of some timedependent Hamiltonian with reflection symmetry $\mathcal{H}(t)=\mathcal{H}(-t)$. We use $\mathcal{H}$ to denote a Liouville space operator which may be any unitary operation or Markovian Lindblad operation. Assume that in addition to the symmetric bins of interest (green bins), the remainder of the time is also divided into bins in a symmetric way so that there is still a reflection symmetry in the bin partitioning also. Now, we permute the bins in the positive side as desired and then make the opposite order in the negative side so that the reflection symmetry is kept. An example of such an operation is shown in Fig. 11(b). Because of the Strang decomposition, we know that the total evolution operator will stay the same under this rearrangement up to third order:

$$
\mathcal{K}_{-\frac{\tau}{2} \rightarrow \frac{\tau}{2}}=\mathcal{T}_{\text {sym }}[\mathcal{K}]_{-\frac{\tau}{2} \rightarrow \frac{\tau}{2}}+O\left[\left(\frac{s}{\hbar}\right)^{3}\right],
$$

where $\mathcal{T}_{\text {sym }}[x]$ stands for evaluation of $x$ after a symmetric reordering.

\section{Symmetric rearrangement theorem}

From Eq. (D11), we see that if the initial state is the same for a system described by $\mathcal{K}$, and for a system described by 
$\mathcal{T}_{\text {sym }}[\mathcal{K}]$, the final state at $t=\tau / 2$ is the same for both systems up to a third-order correction:

$$
\left|\tilde{\rho}\left(\frac{\tau}{2}\right)\right\rangle=\mathcal{T}_{\text {sym }}\left[\left|\tilde{\rho}\left(\frac{\tau}{2}\right)\right\rangle\right]+O\left[\left(\frac{s}{\hbar}\right)^{3}\right] .
$$

Using Eqs. (D8) and (D9), we see that

$$
|\tilde{\rho}(0)\rangle=\frac{\left|\tilde{\rho}\left(\frac{\tau}{2}\right)\right\rangle+\left|\tilde{\rho}\left(-\frac{\tau}{2}\right)\right\rangle}{2}+O\left[\left(\frac{s}{\hbar}\right)^{2}\right],
$$

and because of Eq. (D12), it also holds that

$$
\begin{aligned}
\mathcal{T}_{\text {sym }}[|\tilde{\rho}(0)\rangle] & =|\tilde{\rho}(0)\rangle+O\left[\left(\frac{s}{\hbar}\right)^{2}\right] \\
& =\frac{\left|\tilde{\rho}\left(\frac{\tau}{2}\right)\right\rangle+\left|\tilde{\rho}\left(-\frac{\tau}{2}\right)\right\rangle}{2}+O\left[\left(\frac{s}{\hbar}\right)^{2}\right],
\end{aligned}
$$

using this in Eq. (D10), we get

$$
\delta A_{\mathrm{tot}}=-2 i\langle A| \mathcal{H}\left(t_{1}\right) \frac{\left|\tilde{\rho}\left(\frac{\tau}{2}\right)\right\rangle+\left|\tilde{\rho}\left(-\frac{\tau}{2}\right)\right\rangle}{2} \delta t+O\left[\left(\frac{s}{\hbar}\right)^{3}\right] .
$$

Expression (D15) no longer depends on the position of the time segment but only on its duration and on the value of $\mathcal{H}$. Thus, the SRT states that the expression above also holds for any symmetric rearrangement,

$$
d A_{\text {tot }}=\mathcal{T}_{\text {sym }}\left[d A_{\text {tot }}\right]+O\left[\left(\frac{s}{\hbar}\right)^{3}\right] .
$$

If we replace $A$ by $H_{0}$ and $\mathcal{H}\left(t_{1}\right)$ by $\mathcal{L}_{c}, \mathcal{L}_{h}$, or $\mathcal{H}_{w}$, we immediately get the invariance of heat and work to symmetric rearrangement (up to $s^{3}$ ). If $|\tilde{\rho}[-(\tau / 2)]\rangle$ is the same for all engines, then $|\tilde{\rho}(\tau / 2)\rangle$ is also the same for all engine types up to $O\left(s^{3}\right)$. Consequently, for all stroke engines, the expressions for work and heat are

$$
W=-2 i\left\langle H_{0}\right| \int_{t \in t_{w}} \mathcal{H}_{w}(t) \frac{d t}{\hbar} \frac{\left.\tilde{\rho}\left(\frac{\tau}{2}\right)\right\rangle+\left|\tilde{\rho}\left(-\frac{\tau}{2}\right)\right\rangle}{2}+O\left[\left(\frac{s}{\hbar}\right)^{3}\right],
$$

$$
\begin{aligned}
Q_{c(h)}= & -2 i\left\langle H_{0}\right| \int_{t \in t_{c(h)}} \mathcal{L}_{c(h)}(t) \frac{d t}{\hbar} \frac{\left|\tilde{\rho}\left(\frac{\tau}{2}\right)\right\rangle+\left|\tilde{\rho}\left(-\frac{\tau}{2}\right)\right\rangle}{2} \\
& +O\left[\left(\frac{s}{\hbar}\right)^{3}\right] .
\end{aligned}
$$

Using the identity $|\tilde{\rho}(\tau / 2)\rangle+|\tilde{\rho}[-(\tau / 2)]\rangle=|\tilde{\rho}(t)\rangle+$ $|\tilde{\rho}(-t)\rangle+O\left[(s / \hbar)^{2}\right]$ that follows from Eq. (D13), the integration over time of the energy flows $j_{w}=$ $\left\langle H_{0}\left|(1 / 2 \hbar) \mathcal{H}_{w}\right| \tilde{\rho}(t)\right\rangle \quad$ and $\quad j_{c(h)}=\left\langle H_{0}\left|(1 / \hbar) \mathcal{L}_{c(h)}\right| \tilde{\rho}(t)\right\rangle$ for continuous engines yields expressions (D17) and (D18) once more. This implies that the SRT (D17) and (D18) holds even if the different operations $\mathcal{L}_{c}, \mathcal{L}_{h}$, and $\mathcal{H}_{w}$ overlap with each other.

We emphasize that all the above relations hold for any initial state and not only in the steady state where $|\tilde{\rho}(\tau / 2)\rangle=|\tilde{\rho}[-(\tau / 2)]\rangle$. The physical implication is that in the equivalence regime, different engines are thermodynamically indistinguishable when monitored at the end of each cycle, even when the system is not in its steady state.

[1] R. Alicki, The Quantum Open System as a Model of the Heat Engine, J. Phys. A 12, L103 (1979).

[2] H. Spohn, Entropy Production for Quantum Dynamical Semigroups, J. Math. Phys. (N.Y.) 19, 1227 (1978).

[3] M. Campisi, P. Talkner, and P. Hänggi, Fluctuation Theorem for Arbitrary Open Quantum Systems, Phys. Rev. Lett. 102, 210401 (2009).

[4] M. Campisi, P. Hänggi, and P. Talkner, Colloquium: Quantum Fluctuation Relations: Foundations and Applications, Rev. Mod. Phys. 83, 771 (2011).

[5] H. T. Quan and H. Dong, Quantum Crooks Fluctuation Theorem and Quantum Jarzynski Equality in the Presence of a Reservoir, arXiv:0812.4955.

[6] F. L. Curzon and B. Ahlborn, Efficiency of a Carnot Engine at Maximum Power Output, Am. J. Phys. 43, 22 (1975).

[7] I. Novikov, The Efficiency of Atomic Power Stations (A Review), J. Nucl. Energy 7, 125 (1958).

[8] M. Esposito, K. Lindenberg, and C. Van den Broeck, Universality of Efficiency at Maximum Power, Phys. Rev. Lett. 102, 130602 (2009).

[9] R. Uzdin and R. Kosloff, Universal Features in the Efficiency at Maximal Work of Hot Quantum Otto Engines, Europhys. Lett. 108, 40001 (2014).

[10] P. Salamon, J. D. Nulton, G. Siragusa, T. R. Andersen, and A. Limon, Principles of Control Thermodynamics, Energy 26, 307 (2001).

[11] B. Andresen, Current Trends in Finite-Time Thermodynamics, Angew. Chem., Int. Ed. Engl. 50, 2690 (2011).

[12] R. Kosloff and T. Feldmann, A Discrete Four Stroke Quantum Heat Engine Exploring the Origin of Friction, Phys. Rev. E 65, 055102 (2002).

[13] F. Plastina, A. Alecce, T. J. G. Apollaro, G. Falcone, G. Francica, F. Galve, N. L. Gullo, and R. Zambrini, Irreversible Work and Inner Friction in Quantum Thermodynamic Processes, Phys. Rev. Lett. 113, 260601 (2014).

[14] A. del Campo, J. Goold, and M. Paternostro, More Bang for Your Buck: Super-Adiabatic Quantum Engines, Sci. Rep. 4, 6208 (2014).

[15] R. Uzdin and R. Kosloff, The Multilevel Four-Stroke Swap Engine and Its Environment, New J. Phys. 16, 095003 (2014).

[16] R. Kosloff, A Quantum Mechanical Open System as a Model of a Heat Engine, J. Chem. Phys. 80, 1625 (1984). 
[17] E. Geva and R. Kosloff, A Quantum Mechanical Heat Engine Operating in Finite Time. A Model Consisting of Spin Half Systems as the Working Fluid, J. Chem. Phys. 96, 3054 (1992).

[18] T. Feldmann and R. Kosloff, Performance of Discrete Heat Engines and Heat Pumps in Finite Time, Phys. Rev. E 61, 4774 (2000).

[19] Y. Rezek and R. Kosloff, Irreversible Performance of a Quantum Harmonic Heat Engine, New J. Phys. 8, 83 (2006).

[20] R. Kosloff and A. Levy, Quantum Heat Engines and Refrigerators: Continuous Devices, Annu. Rev. Phys. Chem. 65, 365 (2014).

[21] U. Harbola, S. Rahav, and S. Mukamel, Quantum Heat Engines: A Thermodynamic Analysis of Power and Efficiency, Europhys. Lett. 99, 50005 (2012).

[22] A. E. Allahverdyan, K. Hovhannisyan, and G. Mahler, Optimal Refrigerator, Phys. Rev. E 81, 051129 (2010).

[23] N. Linden, S. Popescu, and P. Skrzypczyk, How Small Can Thermal Machines Be? The Smallest Possible Refrigerator, Phys. Rev. Lett. 105, 130401 (2010).

[24] M. J. Henrich, F. Rempp, and G. Mahler, Quantum Thermodynamic Otto Machines: A Spin-System Approach, Eur. Phys. J. Spec. Top. 151, 157 (2007).

[25] P. Skrzypczyk, A. J. Short, and S. Popescu, Work Extraction and Thermodynamics for Individual Quantum Systems, Nat. Commun. 5, 4185 (2014).

[26] D. Gelbwaser-Klimovsky, R. Alicki, and G. Kurizki, Work and Energy Gain of Heat-Pumped Quantized Amplifiers, Europhys. Lett. 103, 60005 (2013).

[27] M. Kolář, D. Gelbwaser-Klimovsky, R. Alicki, and G. Kurizki, Quantum Bath Refrigeration Towards Absolute Zero: Challenging the Unattainability Principle, Phys. Rev. Lett. 109, 090601 (2012).

[28] R. Alicki, Quantum Thermodynamics: An Example of Two-Level Quantum Machine, Open Syst. Inf. Dyn. 21, 1440002 (2014).

[29] H. T. Quan, Y.x. Liu, C. P. Sun, and F. Nori, Quantum Thermodynamic Cycles and Quantum Heat Engines, Phys. Rev. E 76, 031105 (2007).

[30] J. Roßnagel, O. Abah, F. Schmidt-Kaler, K. Singer, and E. Lutz, Nanoscale Heat Engine Beyond the Carnot Limit, Phys. Rev. Lett. 112, 030602 (2014).

[31] F. Binder, S. Vinjanampathy, K. Modi, and J. Goold, Quantum Thermodynamics of General Quantum Processes, Phys. Rev. E 91, 032119 (2015).

[32] L. A. Correa, J. P. Palao, D. Alonso, and G. Adesso, Quantum-Enhanced Absorption Refrigerators, Sci. Rep. 4, 3949 (2014).

[33] R. Dorner, S. R. Clark, L. Heaney, R. Fazio, J. Goold, and V. Vedral, Extracting Quantum Work Statistics and Fluctuation Theorems by Single-Qubit Interferometry, Phys. Rev. Lett. 110, 230601 (2013).

[34] L. A. Correa, J. P. Palao, G. Adesso, and D. Alonso, Performance Bound for Quantum Absorption Refrigerators, Phys. Rev. E 87, 042131 (2013).

[35] R. Dorner, J. Goold, C. Cormick, M. Paternostro, and V. Vedral, Emergent Thermodynamics in a Quenched Quantum Many-Body System, Phys. Rev. Lett. 109, 160601 (2012).
[36] D. Gelbwaser-Klimovsky, W. Niedenzu, and G. Kurizki, Thermodynamics of Quantum Systems under Dynamical Control, Adv. At. Mol. Opt. Phys. 64, 329 (2015).

[37] A. S. L. Malabarba, A. J. Short, and P. Kammerlander, Clock-Driven Quantum Thermal Engines, New J. Phys. 17, 045027 (2015).

[38] M. Perarnau-Llobet, K. V. Hovhannisyan, M. Huber, P. Skrzypczyk, N. Brunner, and A. Acín, Extractable Work from Correlations, arXiv:1407.7765v2.

[39] D. Segal and A. Nitzan, Molecular Heat Pump, Phys. Rev. E 73, 026109 (2006).

[40] R. S. Whitney, Most Efficient Quantum Thermoelectric at Finite Power Output, Phys. Rev. Lett. 112, 130601 (2014).

[41] M. Horodecki and J. Oppenheim, Fundamental Limitations for Quantum and Nanoscale Thermodynamics, Nat. Commun. 4, 2059 (2013).

[42] L. del Rio, J. Aberg, R. Renner, O. Dahlsten, and V. Vedral, The Thermodynamic Meaning of Negative Entropy, Nature (London) 474, 61 (2011).

[43] J. Gemmer, M. Michel, and G. Mahler, Quantum Thermodynamics (Springer, Berlin/Heidelberg, 2009).

[44] A. Riera, C. Gogolin, and J. Eisert, Thermalization in Nature and on a Quantum Computer, Phys. Rev. Lett. 108, 080402 (2012).

[45] S. Trotzky, Y.-A. Chen, A. Flesch, I. P. McCulloch, U. Schollwöck, J. Eisert, and I. Bloch, Probing the Relaxation Towards Equilibrium in an Isolated Strongly Correlated One-Dimensional Bose Gas, Nat. Phys. 8, 325 (2012).

[46] P. O. Boykin, T. Mor, V. Roychowdhury, F. Vatan, and R. Vrijen, Algorithmic Cooling and Scalable NMR Quantum Computers, Proc. Natl. Acad. Sci. U.S.A. 99, 3388 (2002).

[47] W. S. Bakr, P. M. Preiss, M. Eric Tai, R. Ma, J. Simon, and M. Greiner, Orbital Excitation Blockade and Algorithmic Cooling in Quantum Gases, Nature (London) 480, 500 (2011).

[48] J. Baugh, O. Moussa, C. A. Ryan, A. Nayak, and R. Laflamme, Experimental Implementation of Heat-Bath Algorithmic Cooling Using Solid-State Nuclear Magnetic Resonance, Nature (London) 438, 470 (2005).

[49] L. J. Schulman, T. Mor, and Y. Weinstein, Physical Limits of Heat-Bath Algorithmic Cooling, Phys. Rev. Lett. 94, 120501 (2005).

[50] F. Rempp, M. Michel, and G. Mahler, Cyclic Cooling Algorithm, Phys. Rev. A 76, 032325 (2007).

[51] J. Goold, M. Huber, A. Riera, L. del Rio, and P. Skrzypzyk, The Role of Quantum Information in Thermodynamics-A Topical Review, arXiv:1505.07835.

[52] S. Vinjanampathya and J. Anders, Quantum Thermodynamics, arXiv:1508.06099.

[53] O. Abah, J. Rossnagel, G. Jacob, S. Deffner, F. SchmidtKaler, K. Singer, and E. Lutz, Single-Ion Heat Engine at Maximum Power, Phys. Rev. Lett. 109, 203006 (2012).

[54] A. Dechant, N. Kiesel, and E. Lutz, All-Optical Nanomechanical Heat Engine, Phys. Rev. Lett. 114, 183602 (2015).

[55] K. Zhang, F. Bariani, and P. Meystre, Quantum Optomechanical Heat Engine, Phys. Rev. Lett. 112, 150602 (2014). 
[56] A. Mari and J. Eisert, Cooling by Heating: Very Hot Thermal Light Can Significantly Cool Quantum Systems, Phys. Rev. Lett. 108, 120602 (2012).

[57] D. Venturelli, R. Fazio, and V. Giovannetti, Minimal SelfContained Quantum Refrigeration Machine Based on Four Quantum Dots, Phys. Rev. Lett. 110, 256801 (2013).

[58] M. Lostaglio, K. Korzekwa, D. Jennings, and T. Rudolph, Quantum Coherence, Time-Translation Symmetry, and Thermodynamics, Phys. Rev. X 5, 021001 (2015).

[59] M. Lostaglio, D. Jennings, and T. Rudolph, Description of Quantum Coherence in Thermodynamic Processes Requires Constraints Beyond Free Energy, Nat. Commun. 6, 6383 (2015).

[60] P. Kammerlander and J. Anders, Quantum Measurement and Its Role in Thermodynamics, arXiv:1502.02673.

[61] M. T. Mitchison, M. P. Woods, J. Prior, and M. Huber, Coherence-Assisted Single-Shot Cooling by Quantum Absorption Refrigerators, arXiv:1504.01593.

[62] J. Åberg, Catalytic Coherence, Phys. Rev. Lett. 113, 150402 (2014).

[63] F. C. Binder, S. Vinjanampathy, K. Modi, and J. Goold, Quantacell: Powerful Charging of Quantum Batteries, New J. Phys. 17, 075015 (2015).

[64] K. Korzekwa, M. Lostaglio, J. Oppenheim, and D. Jennings, The Extraction of Work from Quantum Coherence, arXiv:1506.07875.

[65] S. Rahav, U. Harbola, and S. Mukamel, Heat Fluctuations and Coherences in Quantum Heat Engines, Phys. Rev. A 86, 043843 (2012).

[66] M. O. Scully, M. S. Zubairy, G. S. Agarwal, and H. Walther, Extracting Work from a Single Heat Bath via Vanishing Quantum Coherence, Science 299, 862 (2003).

[67] M. O. Scully, K. R. Chapin, K. E. Dorfman, M. Barnabas Kim, and A. Svidzinsky, Quantum Heat Engine Power Can Be Increased by Noise-Induced Coherence, Proc. Natl. Acad. Sci. U.S.A. 108, 15097 (2011).

[68] M. Campisi, J. Pekola, and R. Fazio, Nonequilibrium Fluctuations in Quantum Heat Engines: Theory, Example, and Possible Solid State Experiments, New J. Phys. 17, 035012 (2015).

[69] Other types of engines consist of small variations and a combination of these types.

[70] R. Alicki and M. Fannes, Entanglement Boost for Extractable Work from Ensembles of Quantum Batteries, Phys. Rev. E 87, 042123 (2013).

[71] K. V. Hovhannisyan, M. Perarnau-Llobet, M. Huber, and A. Acín, Entanglement Generation Is Not Necessary for Optimal Work Extraction, Phys. Rev. Lett. 111, 240401 (2013).

[72] M. Campisi, Fluctuation Relation for Quantum Heat Engines and Refrigerators, J. Phys. A 47, 245001 (2014).

[73] G. Gennaro, G. Benenti, and G. Massimo Palma, Entanglement Dynamics and Relaxation in a Few-Qubit System Interacting with Random Collisions, Europhys. Lett. 82, 20006 (2008).

[74] G. Gennaro, G. Benenti, and G. Massimo Palma, Relaxation Due to Random Collisions with a Many-Qudit Environment, Phys. Rev. A 79, 022105 (2009).
[75] T. Rybár, S. N. Filippov, M. Ziman, and V. Bužek, Simulation of Indivisible Qubit Channels in Collision Models, J. Phys. B 45, 154006 (2012).

[76] M. Ziman, P. Štelmachovič, and V. Bužek, Description of Quantum Dynamics of Open Systems Based on Collisionlike Models, Open Syst. Inf. Dyn. 12, 81 (2005).

[77] R. Kosloff, Quantum Thermodynamics: A Dynamical Viewpoint, Entropy 15, 2100 (2013).

[78] J. Anders and V. Giovannetti, Thermodynamics of Discrete Quantum Processes, New J. Phys. 15, 033022 (2013).

[79] H.-P. Breuer and F. Petruccione, Open Quantum Systems (Oxford University Press, Oxford, 2002).

[80] R. Kosloff and T. Feldmann, Optimal Performance of Reciprocating Demagnetization Quantum Refrigerators, Phys. Rev. E 82, 011134 (2010).

[81] This is, of course, not true for the work repository.

[82] A. E. Allahverdyan, K. Hovhannisyan, and G. Mahler, Optimal Refrigerator, Phys. Rev. E 81, 051129 (2010).

[83] H. E. D. Scovil and E. O. Schulz-DuBois, Three-Level Masers as Heat Engines, Phys. Rev. Lett. 2, 262 (1959).

[84] E. Geva and R. Kosloff, The Quantum Heat Engine and Heat Pump: An Irreversible Thermodynamic Analysis of the Three-Level Amplifier, J. Chem. Phys. 104, 7681 (1996).

[85] A. Levy and R. Kosloff, The Local Approach to Quantum Transport May Violate the Second Law of Thermodynamics, Europhys. Lett. 107, 20004 (2014).

[86] G. Lindblad, On the Generators of Quantum Dynamical Semigroups, Commun. Math. Phys. 48, 119 (1976).

[87] V. Gorini, A. Kossakowski, and E. C. G. Sudarshan, Completely Positive Dynamical Semigroup of $n$-Level System, J. Math. Phys. (N.Y.) 17, 821 (1976).

[88] E. B. Davies, Markovian Master Equations, Commun. Math. Phys. 39, 91 (1974).

[89] S. Mukamel, Principles of Nonlinear Optical Spectroscopy (Oxford University Press, New York, 1995), Vol. 29.

[90] H. O. R. N. Roger and R. J. Charles, Topics in Matrix Analysis (Cambridge University Press, Cambridge, England, 1994).

[91] T. F. Havel, Robust Procedures for Converting Among Lindblad, Kraus and Matrix Representations of Quantum Dynamical Semigroups, J. Math. Phys. (N.Y.) 44, 534 (2003).

[92] E. Andersson, J. D. Cresser, and M. J. W. Hall, Finding the Kraus Decomposition from a Master Equation and Vice Versa, J. Mod. Opt. 54, 1695 (2007).

[93] A. Rivas, A. D. K. Plato, S. F. Huelga, and M. B. Plenio, Markovian Master Equations: A Critical Study, New J. Phys. 12, 113032 (2010).

[94] This can be seen by following the derivation in Ref. [79] and using the formalism introduced in Ref. [106].

[95] T. Jahnke and C. Lubich, Error Bounds for Exponential Operator Splittings, BIT Numerical Math. 40, 735 (2000).

[96] M. D. Feit, J. A. Fleck, and A. Steiger, Solution of the Schrödinger Equation by a Spectral Method, J. Comput. Phys. 47, 412 (1982).

[97] H. De Raedt, Product Formula Algorithms for Solving the Time Dependent Schrödinger Equation, Comput. Phys. Rep. 7, 1 (1987). 
[98] S. Blanes and F. Casas, On the Necessity of Negative Coefficients for Operator Splitting Schemes of Order Higher than Two, Applied Numerical Mathematics 54, 23 (2005).

[99] A. E. Allahverdyan, R. Balian, and Th. M. Nieuwenhuizen, Maximal Work Extraction from Finite Quantum Systems, Europhys. Lett. 67, 565 (2004).

[100] For simplicity, we think of a single-particle engine. Thus, entanglement and spin statistics are irrelevant quantum effects. In addition, in the weak system-bath coupling limit, the entanglement to the baths is negligible.

[101] T. Feldmann and R. Kosloff, Quantum Lubrication: Suppression of Friction in a First-Principles Four-Stroke Heat Engine, Phys. Rev. E 73, 025107(R) (2006).

[102] This is very well known in the context of the Zeno effect.

[103] In the Lindblad framework, any thermalization is intrinsically associated with some dephasing. Yet, here we assume an additional controllable dephasing mechanism.

[104] E. Geva and R. Kosloff, The Three-level Quantum Amplifier as a Heat Engine: A Study in Finite-Time Thermodynamics, Phys. Rev. E 49, 3903 (1994).
[105] A. O. Niskanen, Y. Nakamura, and J. P. Pekola, Information Entropic Superconducting Microcooler, Phys. Rev. B 76, 174523 (2007).

[106] S. Machnes and M. B. Penio, Surprising Interactions of Markovian Noise and Coherent Driving, arXiv: 1408.3056v1.

[107] D. A. Lidar, P. Zanardi, and K. Khodjasteh, Distance Bounds on Quantum Dynamics, Phys. Rev. A 78, 012308 (2008).

[108] R. Uzdin and O. Gat, Time-Energy Trade-off in Unambiguous-State-Discrimination Positive Operator-Valued Measures, Phys. Rev. A 88, 052327 (2013).

[109] R. Uzdin, E. Lutz, and R. Kosloff, Purity and Entropy Evolution Speed Limits for Open Quantum Systems, arXiv:1408.1227.

[110] R. Uzdin, Resources Needed for Non-unitary Quantum Operations, J. Phys. A 46, 145302 (2013).

[111] R. Uzdin, U. Günther, S. Rahav, and N. Moiseyev, TimeDependent Hamiltonians with 100\% Evolution Speed Efficiency, J. Phys. A 45, 415304 (2012). 